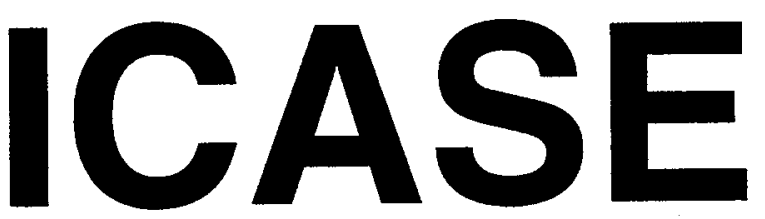

\title{
SHOCK-INDUCED SEPARATED STRUCTURES IN SYMMETRIC CORNER FLOWS
}

\section{Domenic D'Ambrosio \\ Roberto Marsilio}

NASA Contract No. NAS1-19480

December 1995

Institute for Computer Applications in Science and Engineering NASA Langley Research Center

Hampton, VA 23681-0001

Operated by Universities Space Research Association

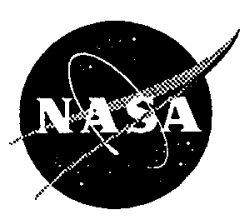

National Aeronautics and Space Administration

Langley Research Center

Hampton, Virginia 23681-0001. 




\title{
Shock-Induced Separated Structures in Symmetric Corner Flows *
}

\author{
Domenic D'Ambrosio \\ Dipartimento di Ingegneria Aeronautica e Spaziale \\ Politecnico di Torino \\ 10129 Torino, Italy \\ Roberto Marsilio \\ Institute for Computer Applications in Science and Engineering \\ NASA Langley Research Center \\ Hampton, VA, 23681, USA.
}

\begin{abstract}
Three-dimensional supersonic viscous laminar flows over symmetric corners are considered in this paper. The characteristic features of such configurations are discussed and an historical survey on the past research work is presented. A new contribution based on a numerical technique that solves the parabolized form of the Navier-Stokes equations is presented. Such a method makes it possible to obtain very detailed descriptions of the flowfield with relatively modest CPU time and memory storage requirements. The numerical approach is based on a space-marching technique, uses a finite volume discretization and an upwind flux-difference splitting scheme (developed for the steady flow equations) for the evaluation of the inviscid fluxes. Second order accuracy is reached following the guidelines of the ENO schemes. Different free-stream conditions and geometrical configurations are considered. Primary and secondary streamwise vortical structures embedded in the boundary layer and originated by the interaction of the latter with shock waves are detected and studied. Computed results are compared with experimental data taken from literature.
\end{abstract}

${ }^{*}$ This research was supported in part by MURST $40 \%$ and it was also supported in part by the National Aeronautics and Space Administration under NASA Contract No. NAS1-19480 while the second author was in residence at the Institute for Computer Applications in Science and Engineering, (ICASE), NASA Langley Research Center, Hampton, VA, 23681 


\section{Introduction}

In the design of supersonic and hypersonic vehicles, the knowledge of the flowfield in the vicinity of interior corners (such as wing-fuselage or fin-fuselage intersections, and box-type supersonic inlets) has a critical importance. In such areas, in fact, complex shock/shock and shock/boundary layer interactions occur, inducing the separation of the viscous layer and its reorganization in vortical structures dominating the shock layer. The most outstanding consequence of such a dramatic change is an important increase in heat fluxes, skin friction coefficients and pressures at the wall in correspondence with the reattachment of the separated flow; transition, shock waves shapes and the efficiency of the air-intakes that might swallow such streams are also affected.

In the last 30 years, several theoretical studies, experimental tests and, more recently, numerical simulations have been carried out in order to investigate the above mentioned configurations. In this paper, the attention will be focused on that part of swept shock waves/viscous layer interactions classified as corner configurations, limited to the laminar regime. A general discussion on the most important features characterizing such flowfields will be proposed and an historical survey of the research conducted in the past will be made.

Finally, a study conducted by the authors using the computational fluid dynamics as an investigative tool will be presented. The numerical method solves the parabolized form of the Navier-Stokes equations: advantages and limitations of such a physical modeling will be discussed and the computational technique will be shortly described. The presence of multiple vortical structures in the shock layer will be demonstrated and their interaction with the wave patterns will be discussed. The effect of the local Reynolds number on the conicity of the flow will be investigated. Moreover, obtained numerical results will be compared, for purpose of validation, with analogous experimental data taken from literature.

\section{Supersonic corner flows}

Supersonic corner flows belong to the family of swept shock wave/viscous layer interactions, that in general can be found in many different forms, but that in the simplest cases are produced by any conical shock generator (not necessarily a wedge) mounted on a flat plate parallel to the flow. If the shock generator apex lies on the leading edge of the plate, the configuration belongs to the intake-type class, whereas, if it is downstream the leading edge, it is called a fin-type configuration. In the former case, the stronger shock wave produced by the generator and the weaker shock due to the flat plate interact and impinge on the viscous layer that is developing on the opposite surface. In the latter case, on the other hand, there is just one shock, since the one produced by the flat plate can be considered as vanishing.

The class of corner flows is usually defined in the literature as a particular case of intaketype configurations, where the shock generator is a wedge and the flat plate is substituted by another wedge. In this case, there are two strong shock waves interacting and impinging on the viscous layers. Depending on whether the leading edges of the wedges are orthogonal to the freestream or not, the corner configuration are called unswept or swept. Moreover, if the two ramps are identical, the corner flow is defined as symmetric; 


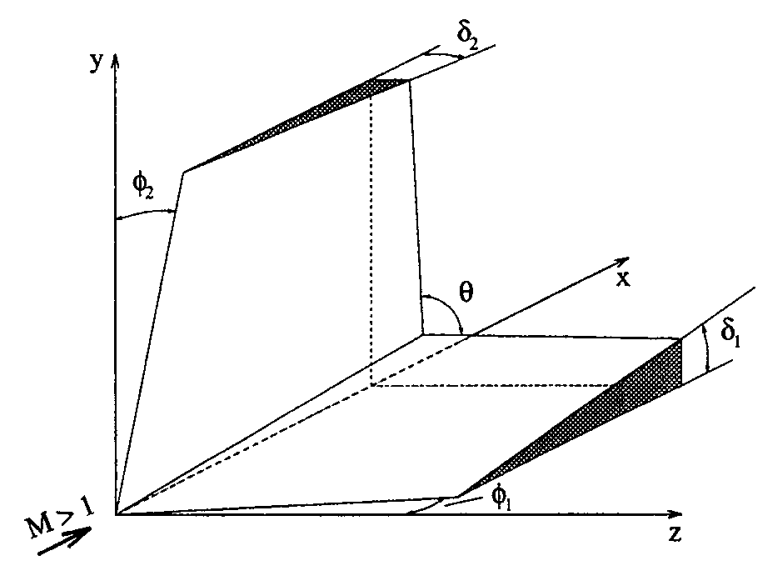

Figure 1: Corner configuration

asymmetric otherwise.

In figure 1, a generic corner configuration is shown and the geometrical parameters that define it are shown. In this paper, the attention will be mostly focused on symmetric unswept corner configurations.

\section{Flowfield structure}

In supersonic corner configurations the presence of the wedges generates two distinct shock waves, whose interference, close to the corner, can produce two different threedimensional shock configurations, called the regular reflection configuration and the Mach disk (or irregular reflection) configuration (figure 2). Both cases are characterized by the fulfillment of the von Neumann conditions [1] at the intersection point (triple point), that consists in imposing, using the Rankine-Hugoniot conditions, the matching of pressure and conical deflection in the unknown high pressure side of the interaction point. The difference between the two configurations is that, in the case of the Mach disk, a further shock is necessary to satisfy the von Neumann conditions. The Mach disk configuration is very common in supersonic corner flows. This fact is seen in figure 3 , taken from reference [2], where the limiting curve separating regular and irregular reflection configurations in the case of symmetric unswept corners and inviscid flows is given in terms of the freestream Mach number and of the wedge angles. It can be seen, for instance, that wedge deflections greater than $5^{\circ}$ generate a Mach disk configuration whatever the freestream Mach number. For completeness, it is necessary to add that, in some occasions, the von Neumann con-
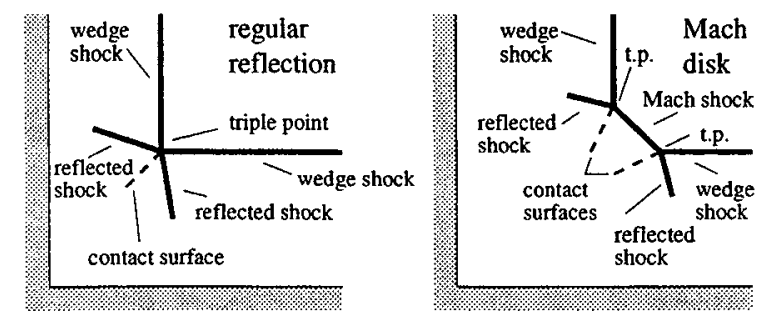

Figure 2: Possible shock interaction configurations 


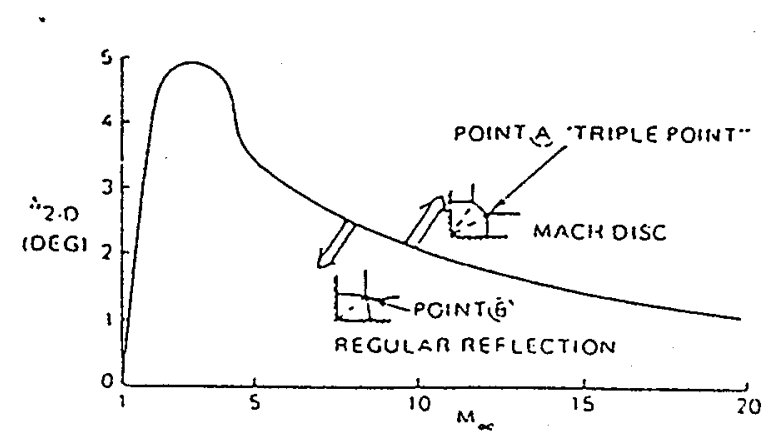

Figure 3: Maximum wedge deflections for regular reflections (symmetric corners) [2]

ditions cannot be satisfied, namely when the Mach number relative to the triple point is decreased below a value dependent on the wedges angles. In this case (a third one), the so called Guderley conditions [1] apply, that consist in inserting a Prandtl-Meyer expansion at the triple point to satisfy the boundary conditions of equal pressure and conical direction. In this last occurrence, however, the shock configuration does not change significantly, except at the triple point [3]. In this paper, only cases where the von Neumann conditions apply will be considered and, in particular, the attention will be concentrated on those situations that are characterized by the Mach disk shock configuration that, as already mentioned, is the most frequent.

The fluid-dynamic pattern typical of the above mentioned flows consists of a system of five shock waves. Two of them, which separate regions I and II in figure 4, are generated by the presence of the wedges; the remaining three are due to the irregular reflection of the previous two. Contact surfaces directed towards the symmetry plane are generated at the triple points because of the different levels of entropy produced by the wave system on either side of the interaction. Shock waves separating regions II and IV impinge on the viscous layer and are reflected as expansion waves from the subsonic part of it. Such expansion fans encounter at their time the slip surfaces and are transformed in compression waves. Additionally, the interaction between the impinging shocks and the viscous layer provokes the separation of the latter in the crosswise direction. Therefore, a streamwise vortex develops, resulting as an obstacle to the crossflow, and thus generating a compression fan analogous to the one typical of two dimensional supersonic flows over a ramp.

Depending on the geometrical and freestream conditions, the vortical structure can be more or less complicated. In particular, with increasing the corner angle $\theta$ and the sweepback angle $\phi$ (see figure 1), the separation is moved inboard and weakened. In both sides of the symmetry plane, it could be constituted of one or two conical sinks and, in the latter case, a secondary vortex below the two sinks may appear. In any case, such separated structures are characterized by the fact that the streamlines that detach from the wall are not those that reattach. Vortical structures heavily affect the pressure and heat-flux distribution at the wall, that reach very high values in correspondence with reattachments. Conversely, a heat-flux trough is usually present where separations occur. It is important to notice that, in laminar regime, the shock-induced separation extends rather far from the corner, affecting regions well outboard with respect to the position of the impinging reflected shock. This feature is a characteristic of the laminar regime; on the contrary, in the turbulent regime, at fixed shock intensity, the separation is less extended, its influence 

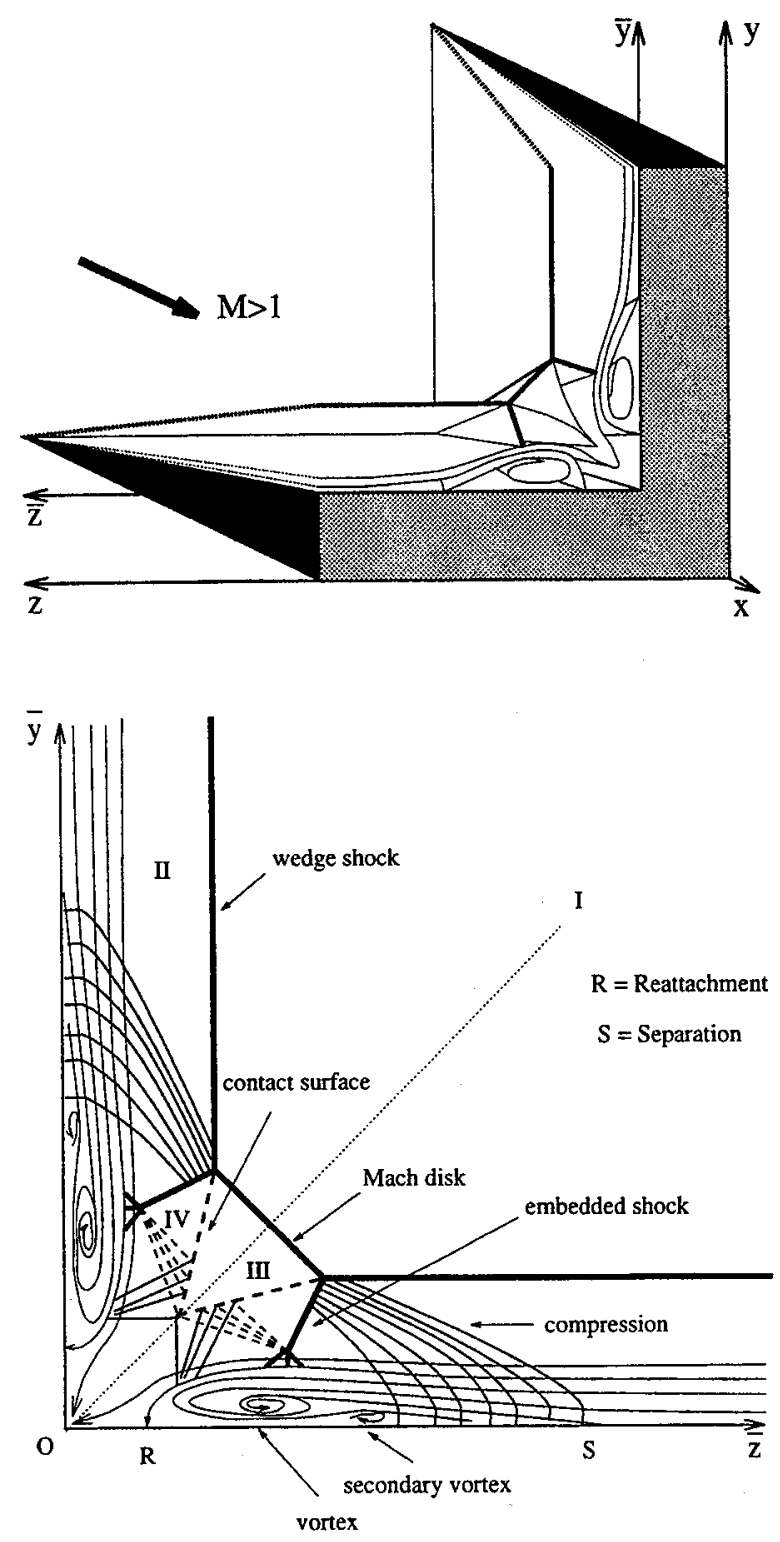

Figure 4: Typical flowfield configuration

being in many cases limited to the vicinity of the embedded shock.

Between the primary vortex reattachment and the corner, a region of constant wall pressure and decreasing heat fluxes appears. This small part of the flowfield, not dominated by viscous/inviscid interactions, can be probably assimilated to a three-dimensional compressible viscous layer, analogous to those studied theoretically at the end of the fifties [4] [5].

The presence of Mach reflections is not limited to corner configurations composed of two wedges, but can easily occur, close to the leading edge, also if the wedges are substituted by two flat plates. It is well known, in fact, that the boundary layer developing on a flat plate appears as a compression surface to the incoming stream and deviates it upwards; in the supersonic regime, this results in the formation of a leading-edge shock wave which potentially is sufficiently bent to give rise to an irregular reflection when interacting with the shock generated by the opposite flat plate; the subsequent flowfield configuration is 


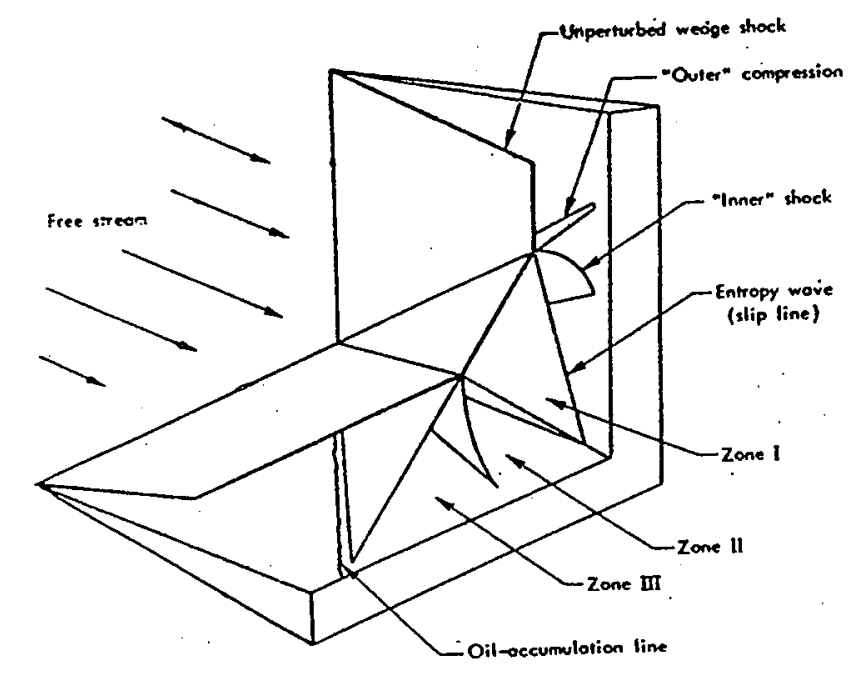

Figure 5: Interpretation of the corner flowfield given in ref.[11]

then analogous to the one described above.

\section{A review of past research}

In the following, a survey on research works concerning corner and intake-type configurations in supersonic and laminar regime will be made. Probably, only a part of the pertinent literature is presented herein. Therefore, apologies are offered in advance for errors of omission.

The first complete research work concerning the problem of shock/viscous layer interaction in a corner was due to Stainback [6] [7], who, between 1960 and 1964, made experimental tests on an intake-type configuration composed of two perpendicular flat plates at Mach 4.95 and 8, in laminar regime. In those years, theoretical investigations on compressible supersonic flows in corners were conducted using boundary layer methods and assuming uniform external flow conditions [4] [5] or considering inviscid solutions [8] [9], but the effect of the interference of these two features was not known. Stainback presented pressure and heat-transfer measurements characterized by peak values noticeably higher than those that could be expected from boundary layer methods. Combining measurements with oil-flow visualizations, he also argued, citing an idea originally due to Bogdonoff and Vas [10], that a shock-induced vortex system was possibly responsible for pressure and heat-transfer variations in the vicinity of the corner.

In the second half of the sixties, following these very first efforts, other experimental tests and, for the first time, a numerical study, were conducted on corner flows. An important contribution was offered by Charwatt and Redekopp, who, in 1967, published an article [11] in which they presented surface flow visualizations, surface pressure measurements and Pitot pressure surveys on symmetric and asymmetric corners at Mach numbers ranging from 2.5 to 4 and in laminar regime. From those data they recognized the Mach reflection shock configuration and also the presence of the compression fan due to the vortex system; nevertheless, they attributed this last feature to inviscid effects (figure 5), interpreting it as a "transmitted' compressive region" and not mentioning the presence of vortices. In any case, their pioneer work was of great importance for the efforts to follow research, 


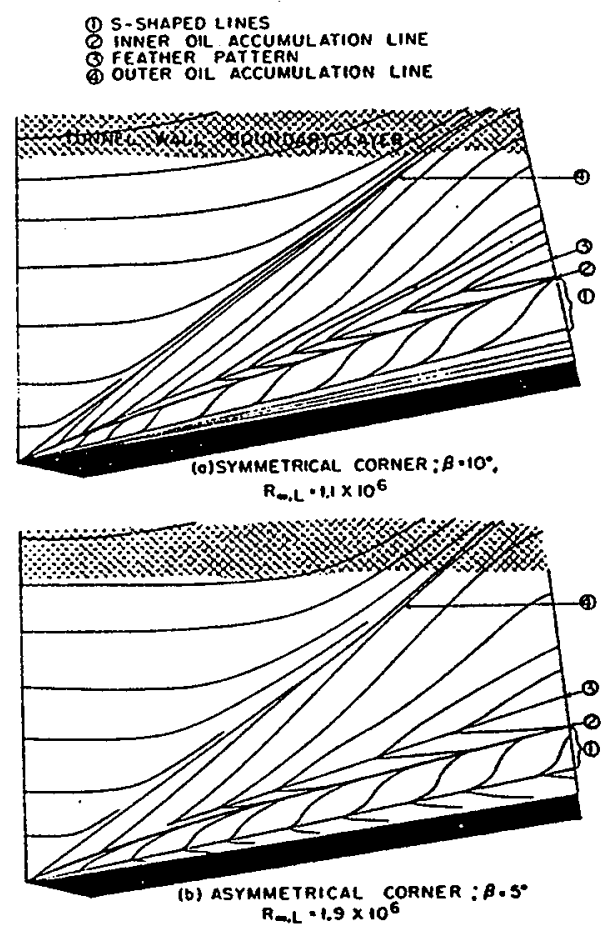

Figure 6: Sketch of the oil-flow patterns visualized in ref.[15]

as it depicted for the first time the corner flow structure. In 1967, Stainback and Weinstein [12], proposing a review of the corner flow problem, stated that a separation and a reattachment were present in the vicinity of the corner owing to the interaction of the shocks system with the viscous layer. They enforced their argument by presenting surface flow visualizations at Mach 8 and flow field visualizations at Mach 20 (in helium) about symmetric and asymmetric corners. In 1969, Cresci et al. [13] showed experimental data and, for the first time, numerical solutions concerning a $90^{\circ}$ corner constituted of two flat plates at Mach 11.2 and in laminar regime. The experiments consisted in surface pressure and heat transfer measurements and in Pitot pressure and total temperature surveys; the computation was performed using a "parabolized" form of the Navier-Stokes equations (a first version of the PNS equations adopted here in the following). The comparison was not quantitative because the computations, for reasons of time, had not been carried out as far downstream as the experiments were performed; however, both experimental and numerical results showed the Mach reflection shock configuration and qualitatively similar patterns.

In the seventies, further experimental research and also extensive numerical studies contributed to increase the knowledge on laminar supersonic corner flows. In 1971, Watson and Weinstein [14] presented results on symmetric corners with various wedge angles $\left(0^{\circ}, 5^{\circ}, 10^{\circ}\right)$ at Mach 20 , in helium and in laminar regime. Correlating Pitot pressure measurements and electron beam flow visualizations with heat-flux measurements and oil-flow visualizations, they clearly recognized the presence of vortices in the flowfield; from surface visualizations, they also noticed the feather-like pattern typical of the secondary separation, but they attributed it to the presence of an embedded shock. The same interpretation was given also by Keyes and Watson [15], who in the same period 


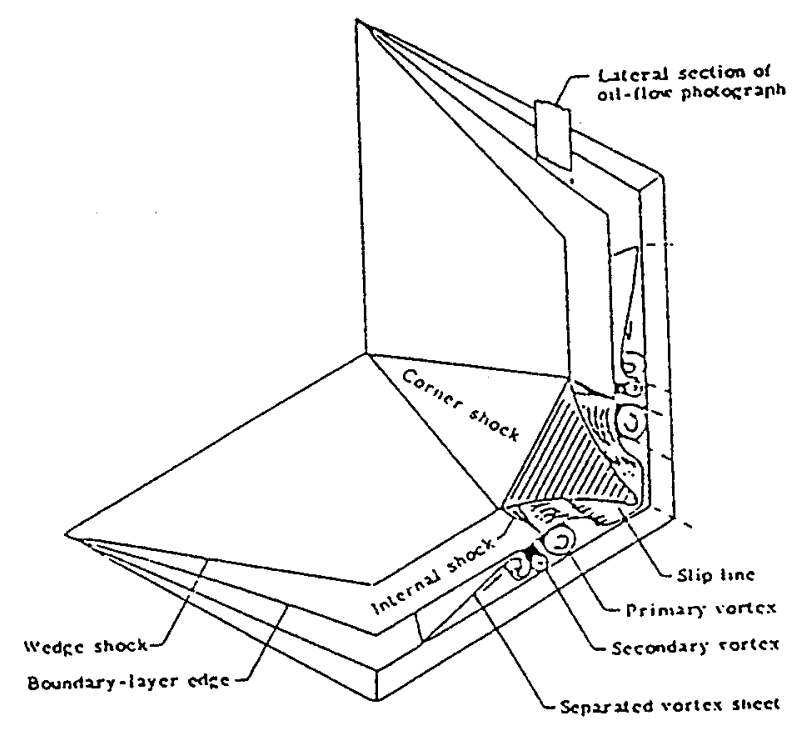

Figure 7: Interpretation of the corner flowfield given in ref.[17]

conducted studies at Mach 19 in helium over symmetric and asymmetric corners (figure 6). In 1971 Korkegi, in his "Survey of viscous interactions associated with high Mach number flight", correlated the presence of peaks and troughs in the heat transfer distribution with the existence of a system of vortices rather than one vortex alone and attributed the appearance of pressure disturbances far from the corner (the compression fan) to separation. In 1972, an article by West and Korkegi [16] showed experimental results on a symmetric corner with wedge angle of $9.5^{\circ}$ at Mach 3 with Reynolds number ranging from $0.4 \times 10^{6}$ to $60 \times 10^{6}$, thus covering both the laminar and turbulent regimes; the authors observed that in the laminar regime the effects of the shock/viscous layer interaction (separation) protract far from the point where the inner shock impinges, while in the turbulent case the separation is limited up to the impingement point area. In 1974, Watson [17] presented the continuation of the work conducted previously by Weinstein and himself [14], including the study of corners with blunted leading edges; from the obtained results he hypothesized the presence of a secondary vortex embedded in the primary one and, perhaps for the first time, he drew a sketch of the vortex system as composed of a separation vortex sheet (which, as the author evidenced, is not the same that reattaches), of a primary vortex where the high speed flow is attracted and of an embedded secondary vortex (figure 7). During the same year, Cooper and Hankey [18] published experimental results obtained considering an intake-type configuration at Mach 12.5 in laminar regime; their aim was to "determine the flowfield structure in a highly asymmetric axial corner and associate areas of elevated heating rates with the accompanying flowfield phenomena". The presence of a separating vortex and of a secondary vortex was evidenced again, though the interpretation of the separation pattern was uncorrect (figure 8). The authors also focused the attention on the inviscid flowfield, showing and discussing the presence of a single triple point. In 1975, Kipke and Hummel [19] published results concerning extensive studies on unswept corner configurations in laminar regime that had been conducted at the TU Braunshweig. Many experiments had been performed at Mach 


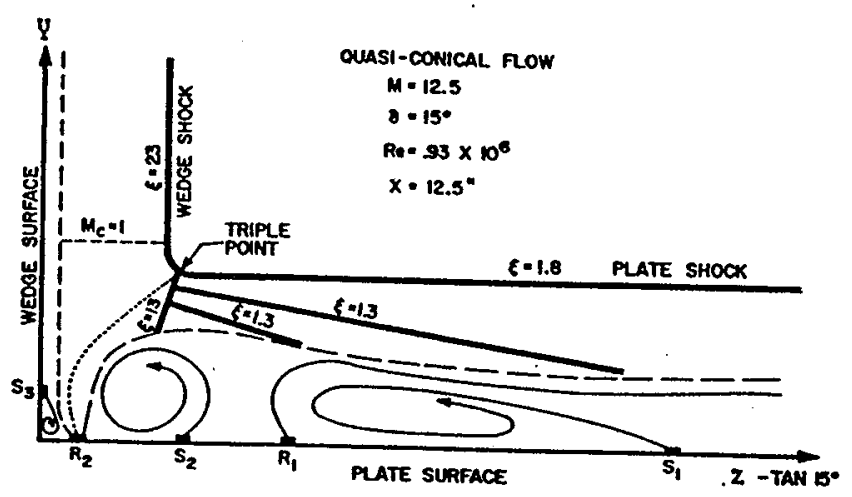

Figure 8: Interpretation of the intake-type configuration flowfield given in ref.[18]

12.3 and 16 varying the wedges angle and the corner angle, demonstrating that the separation intensity (and therefore heat transfer and pressure peaks in correspondence with the reattachment) increases with decreasing the corner angle. Moreover, a very detailed description of the flowfield was given correlating pitot-pressure surveys, oil-flow visualizations and heat-transfer and wall pressure distributions. In 1976, Korkegi published a study [20] on the existing experimental data on three-dimensional skewed shock wave interactions with both laminar and turbulent viscous layers. After having pointed out that extensive shock-induced separation is possible also for turbulent flows, but needs larger shock intensities with respect to the laminar regime, he described the general structure of three-dimensional shock-induced separated flow regions starting from unseparated flows up to extensive separated flows.

In the middle of the seventies, numerical studies were conducted by Kutler [21], Shankar et al. [22] and Anderson and Nangia [23], who all solved the conical Euler equations using shock capturing techniques. Kutler [21], in particular, simulated the freestream conditions of the West and Korgegi's tests [16], obtaining a satisfying agreement with the highest Reynolds number experiments. In 1977, for the first time, Shang and Hankey [24] published numerical results on a supersonic intake-type configuration that had been obtained solving the full Navier-Stokes equations using a time-dependent shock capturing technique. They reproduced Cooper and Hankey's results [18], obtaining a good agreement with surface experimental measurements and confirming the presence of only one triple point in the flowfield but, owing to the coarseness of the grid used (the fine one was $8 \times 32 \times 36$ ), the thickness of the viscous layer and thus the distance of the shock wave system from the wall was underestimated. For the same reason, they did not capture the secondary vortex, though its presence could be perceived from surface data; the authors argued that the secondary separation could be caused by an embedded supersonic region (created by the primary vortex) followed by a compression shock. In 1978, Hung and MacCormack [25] were the first ones to obtain a numerical solution of viscous/inviscid interactions for fully turbulent flows. The year after, Shang, Hankey and Petty [26] reproduced numerically the West and Korkegi's [16] experiments including a transition model in their code. In 1980, Marconi [2][3] solved the conical Euler equations using a shock fitting technique and compared his results with Kutler [21], West and Korkegi[16] and Charwatt and Redekopp[11].

During the eighties, the production of studies concerning supersonic laminar corner flows was rather scarce. Nevertheless, in 1984, results of a new extensive campaign performed at 


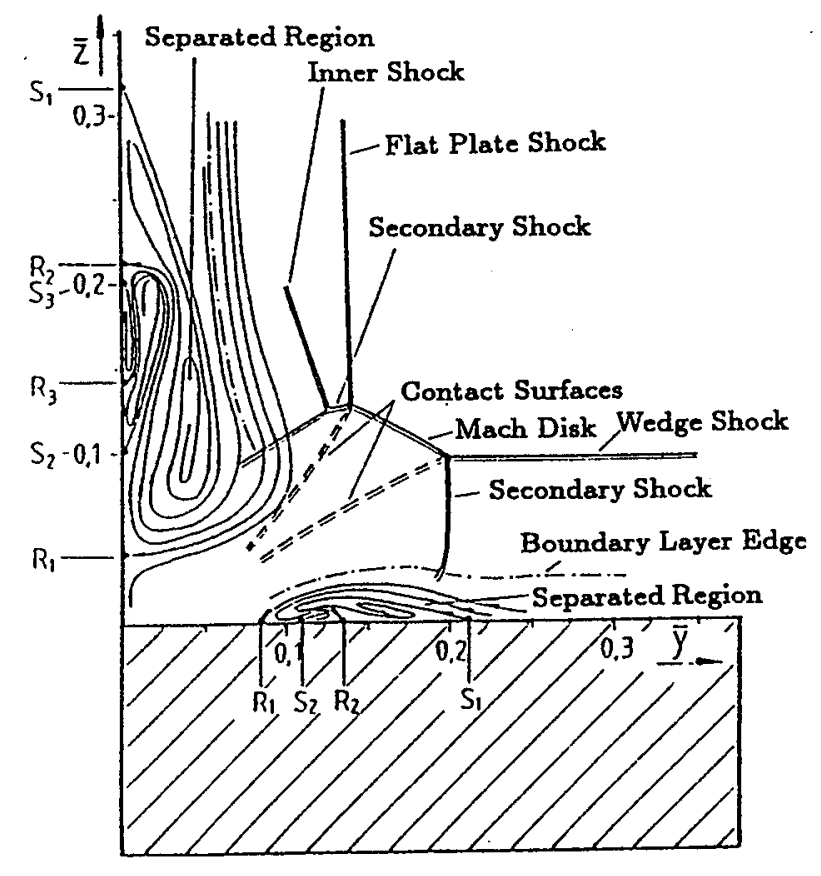

Figure 9: Interpretation of the corner flowfield given in ref.[31]

the TU Braunshweig were published by Möllenstädt[27]. Experiments were conducted at Mach 12.3 and in laminar regime on swept symmetric corner configurations with different sweep angles and corner angles and showed that, with increasing leading edge sweep, the shock system is displaced towards the corner and the vortex system is less intense. Such results were republished in 1987 by Hummel [28], together with those by Kipke and Hummel [19] previously cited.

In 1991, Qin, Scriba and Richards [29] showed numerical results obtained solving the locally conical Navier-Stokes equations and reproducing the experiments performed by Möllenstädt [27]. A peculiarity of such results is that an additional couple of vortices was captured close to the corner. In 1992, experimental investigations, described by Degrez in reference [30], were conducted at the Von Karman Institute for Fluid Dynamics on intake-type configurations at Mach 2 and Mach 6 in laminar regime. There, perhaps for the first time, tertiary separation was observed in the Mach 6 case with wedge angles greater than $6^{\circ}$. During the same year, Petzel and Hummel [31] presented experimental results concerning an intake-type configuration with a $8^{\circ}$ wedge at Mach 12.6 in laminar regime, showing the presence of a tertiary vortex on the flat plate surface (figure 9 ). In 1991, Marsilio started numerical investigations on inviscid corner configurations at the Politecnico di Torino. He solved the steady-state three-dimensional Euler equations using a space-marching upwind finite volumes method. In his paper of 1993 [32], he showed that, if the shocks intensities were sufficiently large, the contact surfaces tend to roll up, generating two spiral singularities. The most outstanding feature, however, was that such vortices, initially symmetric, are not stable and move to an asymmetric configuration, which is stable. To verify the reliability of such results, comparisons were made with other numerical experiments performed at the VKI solving the time-dependent conical Euler equations: the resulting conclusions, published in [33] by Degrez et al., showed 


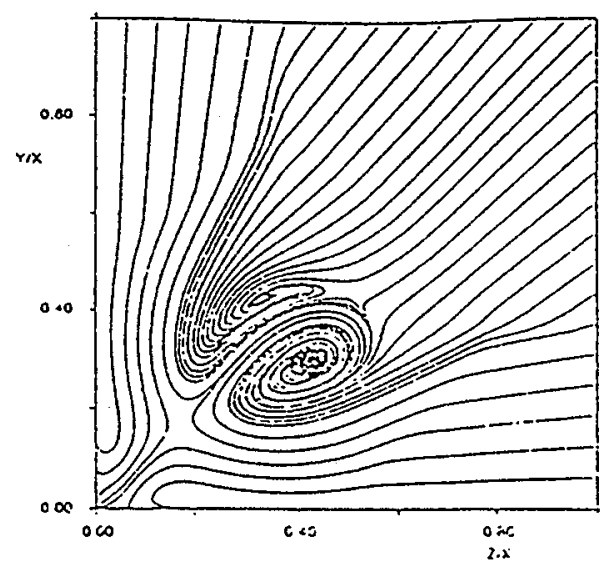

(a) Conical compulation

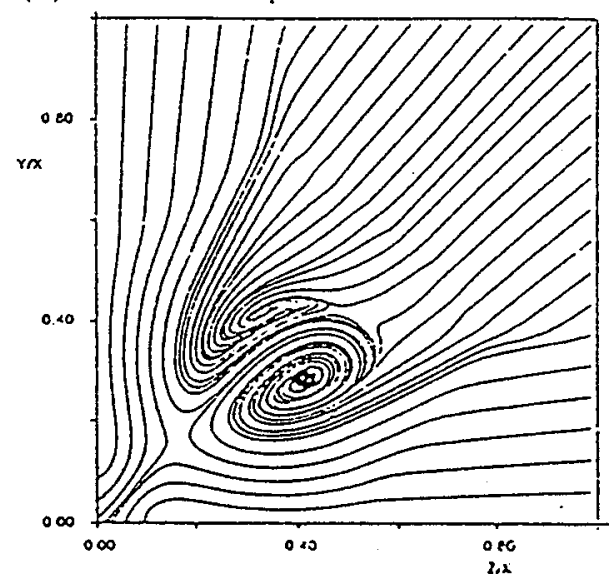

(b) Space-marching computation

Figure 10: Comparison performed in ref.[33]

that asymmetric vortical solutions appear independently of the computational method used (figure 10), but are very sensitive to numerical dissipation.

\section{Present contribution: methodology}

The previously cited research work on inviscid supersonic corner flows initiated by R. Marsilio has been continued and has found its natural evolution in the investigation of viscous flows. Now, the only completely correct way of solving numerically three-dimensional compressible viscous flows is to integrate in time the full Navier-Stokes equations until a steady-state (if existent) is reached. This approach is affordable with current computer capabilities, but, in particular if many grid points are needed to solve in detail complex fluid-dynamic features, it could be excessively CPU-time and memory requiring and thus, in practice, unaffordable. In the case of supersonic steady-state flows, however, this practical difficulty can be partially circumvented with the aid of the approximate form of the full Navier-Stokes equations known as Parabolized Navier-Stokes equations.

The advantage brought by the Parabolized Navier-Stokes (PNS) equations is that they can be solved using a space-marching technique, a characteristic which allows to spend 
relatively short computation times and permits noticeable memory savings (if compared with a time dependent method). Therefore, it is possible to reinvest CPU-time and memory in more refined grids, thus permitting a very neat resolution of the flowfield. As a drawback, the parabolizing assumption requires the freestream Mach number to be supersonic and the streamwise velocity always positive; thus, streamwise flow separations are excluded, but crossflow separations are permitted. Moreover, the streamwise pressure gradient is altered in the subsonic part of the flowfield [34], this fact implying that in that region the contribution of the backward travelling signals is neglected.

In this investigation, the three-dimensional Parabolized Navier-Stokes equations are used. Such a formulation is derived from the steady-state full Navier-Stokes equations with the aim of obtaining a system of equations representing a well posed problem with respect to an integration performed using a space-marching technique. At this point, any derivative in the streamwise direction contained in the stress tensor is neglected, all viscous and heat fluxes in the streamwise direction are dropped and the pressure gradient in the subsonic layer is properly altered. With such modifications, valid only for sufficiently high Reynolds numbers, the Navier-Stokes equations are reduced to a set of hyperbolicparabolic equations [34][35].

The full Navier-Stokes equations can be written in integral conservative form in the following way:

$$
\frac{\partial}{\partial t} \int_{\mathcal{V}} \mathbf{W} d \mathcal{V}+\int_{\mathcal{S}} \mathbf{F}_{\mathbf{I}} \cdot \vec{n} d \mathcal{S}+\int_{\mathcal{S}} \mathbf{F}_{\mathbf{V}} \cdot \vec{n} d \mathcal{S}=0
$$

where $\mathcal{V}$ represents an arbitrary volume inclosed in a surface $\mathcal{S}$ with unit normal $\vec{n}$ positive if directed outward.

System (1) can be reduced to non-dimensional form with the help of the following reference values: $L$ for length, $\rho_{\infty}$ for density, $T_{\infty}$ for temperature, $\sqrt{R T_{\infty}}$ for velocity, $R T_{\infty}$ for energy per unit mass and $\mu\left(T_{\infty}\right)$ for viscosity. Therefore, from now on, the flowfield variables should be considered as non-dimensional. In particular, $\mathbf{W}$ is the hypervector of conservative variables, tensor $\mathbf{F}_{\mathbf{I}}$ contains the inviscid fluxes and tensor $\mathbf{F}_{\mathbf{V}}$ contains the viscous fluxes:

$$
\begin{aligned}
\mathbf{W} & =\{\rho, \rho \vec{q}, E\}^{T} \\
\mathbf{F}_{\mathbf{I}} & =\{\rho \vec{q}, p \bar{I}+\rho \vec{q} \otimes \vec{q},(E+p) \vec{q}\}^{T} \\
\mathbf{F}_{\mathbf{V}} & =\frac{\sqrt{\gamma} M_{\infty}}{R e_{\infty}}\{\overrightarrow{0},-\overline{\bar{\tau}} ;-k \nabla T-\overline{\bar{\tau}} \cdot \vec{q}\}^{T}
\end{aligned}
$$

Quantities $\rho, p$ and $\vec{q}=\{u, v, w\}^{T}$ are respectively the local density, pressure and velocity; $E$ represents the total energy per unit volume:

$$
E=\rho\left(e+\frac{|\vec{q}|^{2}}{2}\right)
$$

$e$ is the internal energy per unit mass, $M_{\infty}$ and $R e_{\infty}$ are the freestream Mach number and Reynolds number, $\gamma$ is the ratio of the specific heats and, finally, $\bar{I}$ is the unit matrix. Viscous stresses are contained in tensor $\overline{\bar{\tau}}$, with

$$
\tau_{i j}=\mu\left[\left(\frac{\partial q_{j}}{\partial x_{i}}+\frac{\partial q_{i}}{\partial x_{j}}\right)-\frac{2}{3}(\nabla \vec{q}) \delta_{i j}\right]
$$


where $\delta_{i j}$ is the Kröneker's symbol. The viscosity is calculated via Sutherland's law:

$$
\mu=T^{3 / 2}\left(\frac{1+T_{r e f}}{T+T_{r e f}}\right)
$$

where

$$
T_{r e f}=\frac{110.4 K}{T_{\infty}}
$$

and the thermal conductivity $k$ is obtained according to the relation:

$$
k=\frac{\mu}{\operatorname{Pr}} \frac{\gamma}{\gamma-1}
$$

where $\operatorname{Pr}$ is the Prandtl number. Finally, the perfect gas relationship completes the set of equations

$$
\frac{p}{\rho}=T
$$

In the parabolizing assumption, assuming the streamlines direction to be fairly close to the $\mathrm{x}$-axis, the components of the stress tensor necessary to evaluate the viscous fluxes are reduced to the following form:

$$
\begin{aligned}
\tau_{x x}^{*} & =-\frac{2}{3} \mu\left(v_{y}+w_{z}\right) \\
\tau_{y y}^{*} & =\frac{2}{3} \mu\left(2 v_{y}-w_{z}\right) \\
\tau_{z z}^{*} & =\frac{2}{3} \mu\left(2 w_{z}-v_{y}\right) \\
\tau_{x y}^{*} & =\tau_{y x}^{*}=\mu u_{y} \\
\tau_{x z}^{*} & =\tau_{z x}^{*}=\mu u_{z} \\
\tau_{y z}^{*} & =\tau_{z y}^{*}=\mu\left(v_{z}+w_{y}\right)
\end{aligned}
$$

Moreover, the $\mathrm{x}$-component of the temperature gradient is neglected:

$$
\nabla T^{*}=T_{y} \vec{j}+T_{z} \vec{k}
$$

The streamwise pressure gradient is splitted according to the technique suggested by Vigneron, Rakich and Tannehill in reference [34]:

$$
p_{x}=\omega p_{x}+(1-\omega) p_{x}
$$

If only the first term of the RHS of equation (18) is retained and the second term is considered as a source term, the set of equations containing only the convective terms is hyperbolic, provided that

$$
\begin{cases}u>0 & \\ \omega<\frac{\gamma M_{x}^{2}}{1+(\gamma-1) M_{x}^{2}} & \text { for } \quad M_{x}<1 \\ \omega=1 & \text { for } \quad M_{x} \geq 1\end{cases}
$$


where $M_{x}$ is the local Mach number in the $x$-direction. The first condition contained in equations (19) prevents the PNS approximation to be used when the flow separates in the streamwise direction. A short analysis of the second condition shows that $\omega$ is equal to 1 when $M_{x}=1$ and is null when $M_{x}=0$; this means that the effect of the streamwise pressure gradient is completely neglected at the wall and is more and more considered as the flow approaches supersonicity. For $M_{x}$ greater than 1 the effect of $p_{x}$ will be entirely taken into account and the value of $\omega$ will be unity. Of course, since a space-marching integration is desired, the freestream Mach number will necessarily have to be supersonic. Combining the cited assumptions, system (1) is finally reduced to the following form:

$$
\int_{\mathcal{S}} \mathbf{F}_{\mathbf{I}}^{*} \cdot \vec{n} d \mathcal{S}+\int_{\mathcal{S}} \mathbf{F}_{\mathbf{V}}^{*} \cdot \overrightarrow{n^{*}} d \mathcal{S}=\int_{\mathcal{S}} \mathbf{P} \cdot \vec{n} d \mathcal{S}+\mathbf{P}^{\prime}
$$

where

$$
\begin{aligned}
\mathbf{F}_{\mathbf{I}}^{*} & =\left\{\rho \vec{q}, p \overline{I^{*}}+\rho \vec{q} \otimes \vec{q},(E+p) \vec{q}\right\}^{T} \\
\mathbf{F}_{\mathbf{V}}^{*} & =\frac{\sqrt{\gamma} M_{\infty}}{R e_{\infty}}\left\{\overrightarrow{0},-\overline{\overline{\tau^{*}}},-k \nabla T^{*}-\overline{\overline{\tau^{*}}} \cdot \vec{q}\right\}^{T} \\
\mathbf{P} & =\left\{\overrightarrow{0},-(1-\omega) p \overline{I^{* *}}, \overrightarrow{0}\right\}^{T}
\end{aligned}
$$

and $\overline{I^{*}}$ and $\overline{I^{* *}}$ are scalar matrices with:

$$
\begin{aligned}
\operatorname{diag} \overline{I^{*}} & =(\omega, 1,1) \\
\operatorname{diag} \overline{I^{* *}} & =(1,0,0)
\end{aligned}
$$

Since viscous and heat fluxes in the steamwise direction are dropped, vector $\overrightarrow{n^{*}}$ contains only the components in the $\mathrm{y}$-direction and in the $\mathrm{z}$-direction of the normal unit vector, that is $\overrightarrow{n^{*}}=\left(0, n_{y}, n_{z}\right)$.

Source term $\mathbf{P}^{\prime}$ must be added to make the integral formulation coherent with equation (18), as noticed in references [36] and [37]. In fact, the corresponding integral form of $\omega p_{x}$ is:

$$
\omega \int p n_{x} d \mathcal{S}=\int \omega p n_{x} d \mathcal{S}-p \int \omega n_{x} d \mathcal{S}
$$

Therefore, we have:

$$
\mathbf{P}^{\prime}=\{\overrightarrow{0}, \bar{\Omega}, \overrightarrow{0}\}^{T}
$$

where $\bar{\Omega}$ is a scalar matrix with

$$
\operatorname{diag} \bar{\Omega}=\left(p \int \omega n_{x} d \mathcal{S}, 0,0\right)
$$

In the numerical approach presented here, explained in detail in reference [38], governing equations are integrated in an explicit fashion and the physical domain is discretized according to a finite volume approach. The convective part of the equations (inviscid fluxes) is treated using a flux-difference-splitting technique with an approximate solution of a Riemann problem at each cell interface conceived for steady flows [39], while diffusive terms (viscous fluxes) are calculated using a centered scheme and exploiting the Gauss theorem in a discrete form. A second order accuracy is reached following the guidelines of the Essentially Non Oscillatory schemes [40] with a properly limited linear reconstruction of the solution inside each cell and at each step of integration. Presently, only inert gases in laminar regime are considered, but the future addition of thermochemical or turbulence models is certainly feasible. 


\section{Present contribution: results}

Unswept symmetric corner configurations have been investigated simulating the same geometrical and freestream conditions that characterize some of the experimental results obtained by Kipke and Hummel and presented in references [19] and [28]. In particular, comparisons have been carried out in the following cases (see also figure 1 for the interpretation of the symbols):

\begin{tabular}{|c|c|c|c|c|c|c|}
\hline$\#$ & $\delta$ & $\theta$ & $M_{\infty}$ & $R e_{\infty} / m$ & $T_{\infty}[\mathrm{K}]$ & $T_{w}[\mathrm{~K}]$ \\
\hline 1 & $8^{\circ}$ & $90^{\circ}$ & 12.3 & $5 \times 10^{6}$ & 45.3 & 300 \\
\hline 2 & $8^{\circ}$ & $90^{\circ}$ & 16.0 & $1.7 \times 10^{6}$ & 25.9 & 300 \\
\hline 3 & $8^{\circ}$ & $120^{\circ}$ & 16.0 & $1.7 \times 10^{6}$ & 25.9 & 300 \\
\hline
\end{tabular}

with a Prandtl number of 0.72 .

The geometry of case \#1 has also been used to perform some further numerical experiments, that will be shown in the following sections.

\subsection{Salient features and comparisons with experiments}

The first results to be presented are related to test case \#1. The grid used was composed of $100 \times 100$ cells, with about 50 cell $\mathrm{s}$ in the normal direction inside the shock layer. A general idea of the computed flowfield can be perceived from figure 11, where crossflow streamlines, static pressure contours, Mach number contours and stagnation temperature contours corresponding to a local Reynolds number $R e_{x}=450000(\mathrm{x}=0.09 \mathrm{~m})$ have been plotted. Crossflow streamlines are obtained projecting the velocity vectors on a sphere centered at the intersection of the wedges leading edges; such a representation makes it possible to visualize the crossflow structure. Observing figure $11 \mathrm{a}$, it is possible to notice the presence of the vortex system that characterize supersonic corner configurations; such a separated structure will be analyzed in greater detail further on. The related wave system is clearly shown in figure $11 \mathrm{~b}$, where it is possible to appreciate the Mach reflection, the compression fan generated by the presence of the separated structure and the reflection of the impinging shock on the viscous layer. The latter interaction results in an expansion fan that, interfering with the slip surfaces, generates a compression wave. In the vicinity of the corner, a region of high but constant pressure is present. The Mach number contours drawn in figure 11c add to the previous pictures the slip surfaces and show the viscous layer thickness; it must be stressed that the subsonic region is very thin, as demonstrated by the fact that the contour corresponding to Mach 1 is almost undistinguishable from the wall. Finally, the stagnation temperature contours are shown to demonstrate that the flow that reattaches owns almost the whole total enthalpy of the freestream, since the low energy flow coming from the viscous layer is entirely swallowed by the vortical structures. In figure $12 \mathrm{a}$, the vortical structure that appeared in figure 11a has been enlarged. It is possible to see a primary separated structure composed of two foc $i$ and a simple secondary vortex. The topological scheme is shown in figure $12 \mathrm{~b}$, that should be compared with figure 12c. Particles belonging to the slowest part of the boundary layer are captured by the right-hand side focus $\left(F_{1 A}\right)$ of the primary separation, that starts in $s_{1}$; this happens up to a certain distance from the wall: continuing to move apart, faster particles are encountered, that are captured by the left-hand side focus $\left(F_{1 B}\right)$ of the primary separation; 

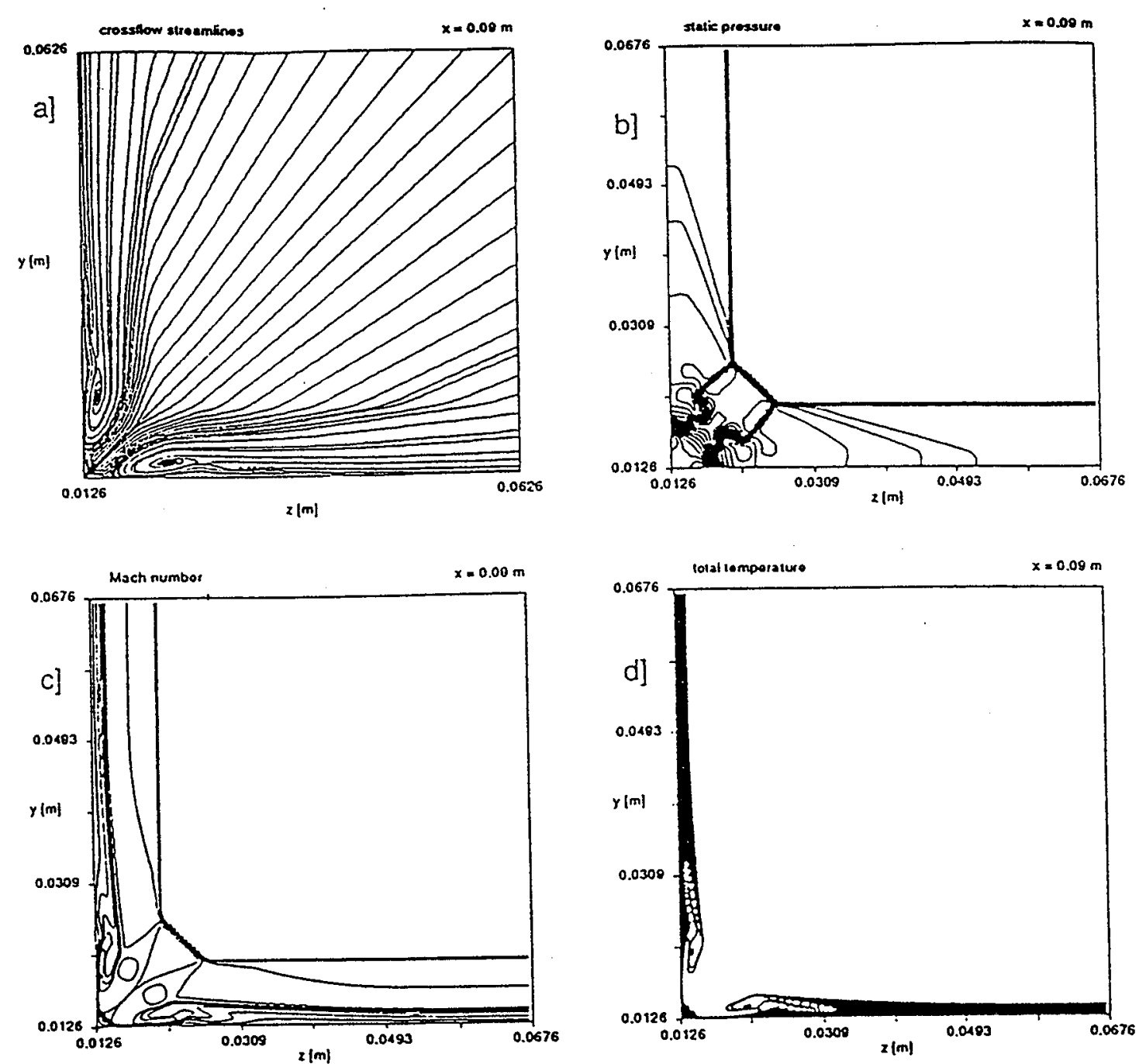

Figure 11: Computed a)crossflow streamlines, b)static pressure contours, c)Mach number contours and d)total temperature contours for test case \#1.

the entire viscous layer is thus swallowed by the vortical structure. Proceeding further upwards, streamlines belonging to the inviscid part of the shock layer are captured again by focus $F_{1 A}$; therefore, a saddle must exist between the two foci. Then, a small amount of particles is entrained in the secondary vortex, whose focus is $F_{2}$ and that is also defined by the separation and reattachment lines $s_{2}$ and $r_{2}$ (see also the zoomed view in figure 12d). Finally, a streamline reattaches in $r_{1}$, closing in this way the primary separation; such particles are coming from the inviscid part of the flowfield, and thus own a great amount of energy: it is for this reason that, when they stop at the stagnation point, they increase noticeably their temperature, provoking an intense heat flux towards the cold wall. Streamlines above the reattaching one are deviated towards the corner and appear as a supersonic jet; in this region it is possible to appreciate other singularities, that will be considered in a following section. By now, it is important to notice that the separated structure just described is topologically correct. In fact, it is composed of four half-saddles (separations and reattachments), three foci (two belonging to the primary separation and one to the secondary vortex) and one saddle (between $F_{1 A}$ and $F_{1 B}$ ). Attributing a positive unit value to each focus and a negative unit value to each saddle [41], the total gives zero, that means that the considered structure is self-consistent. 

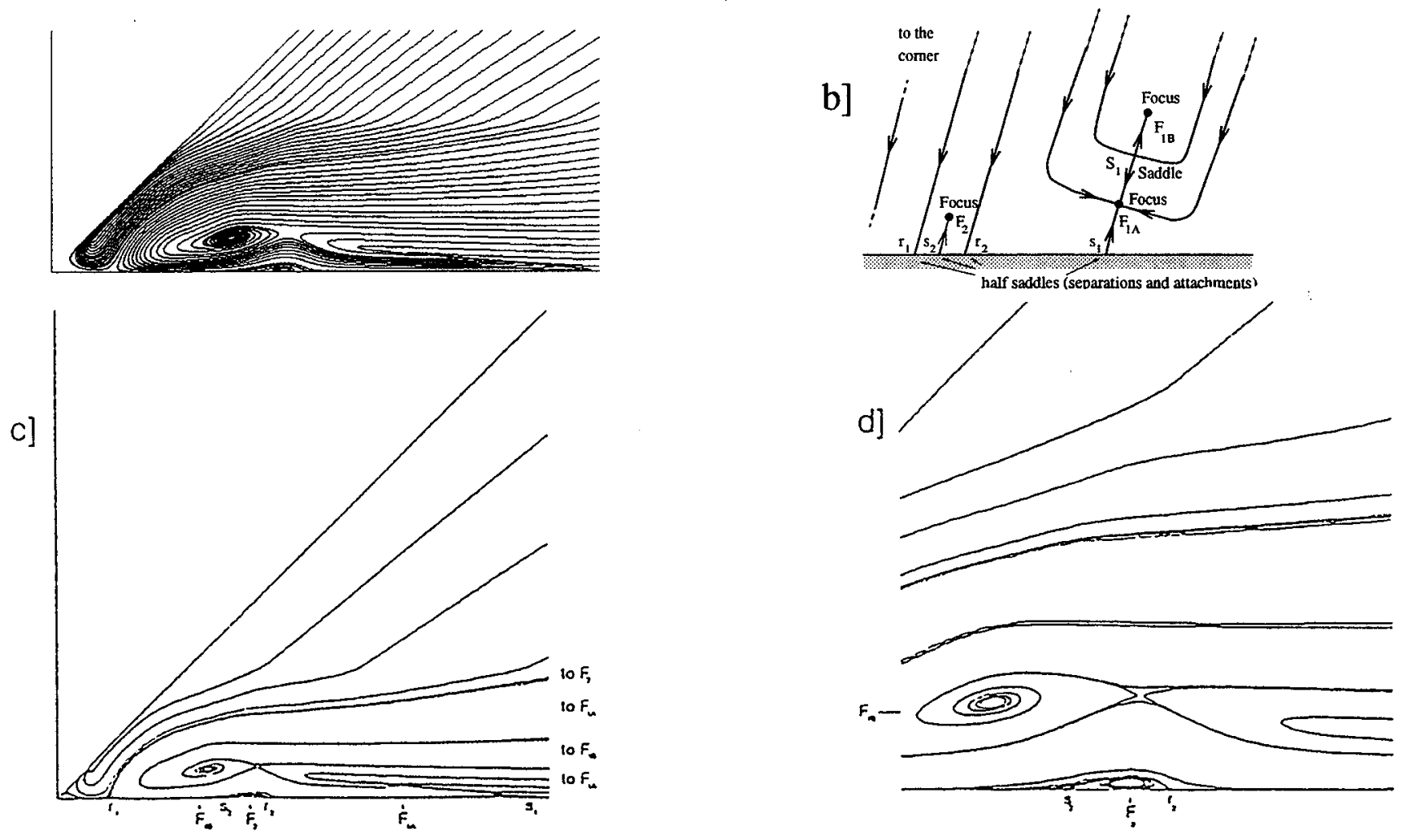

Figure 12: a)computed crossflow streamlines, b)topology of the separated structure, c)limiting crossflow streamlines, d)zoom of the saddle point region.

Up to now, only results concerning a particular cross section have been presented, but nothing has been shown to give a general idea of the evolution of the flow along the corner. Figure 13 fills such a gap, displaying the limiting streamlines at the wall, which are the numerical equivalent of oil-flow visualizations; crossflow streamlines have been placed beside for a better comprehension of the picture. The separation and reattachment of the primary vortex are well in evidence, and also the presence of the secondary vortex at the wall can be perceived. It is possible to notice that streamlines are deviated before reaching the separation line, thus generating an upstream influence line that is the forwardmost extent of the interaction. It should be noted that the presence of the vortical structures and of the related compression fan transports the three-dimensional effects due to the corner at noticeable distances from it inside the shock layer, making it necessary to fix rather ample external boundaries. If a square or rectangular grid is used, this results in having a lot of points outside the shock layer.' To avoid to waste CPU-time in computing points for which it is known a priori that the freestream conditions apply, a procedure has been implemented that detects the position of the shock layer at each step of integration and solves the flowfield only in the part of the domain containing it.

The same computed results presented above are now compared with analogous experimental data extracted from reference [28]. In figure 14, Pitot pressure contours corresponding to a distance from the leading edge of $0.09 \mathrm{~m}$ are shown. The contours shapes fit well, and also the qualitative agreement is quite good. In fact, the wedge-shock position is not exactly the same, as the computed one corresponds to a local slope of about $4.9^{\circ}$ with respect to the wedge, versus an angle of about $5.5^{\circ}$ for the experiments (the inviscid 

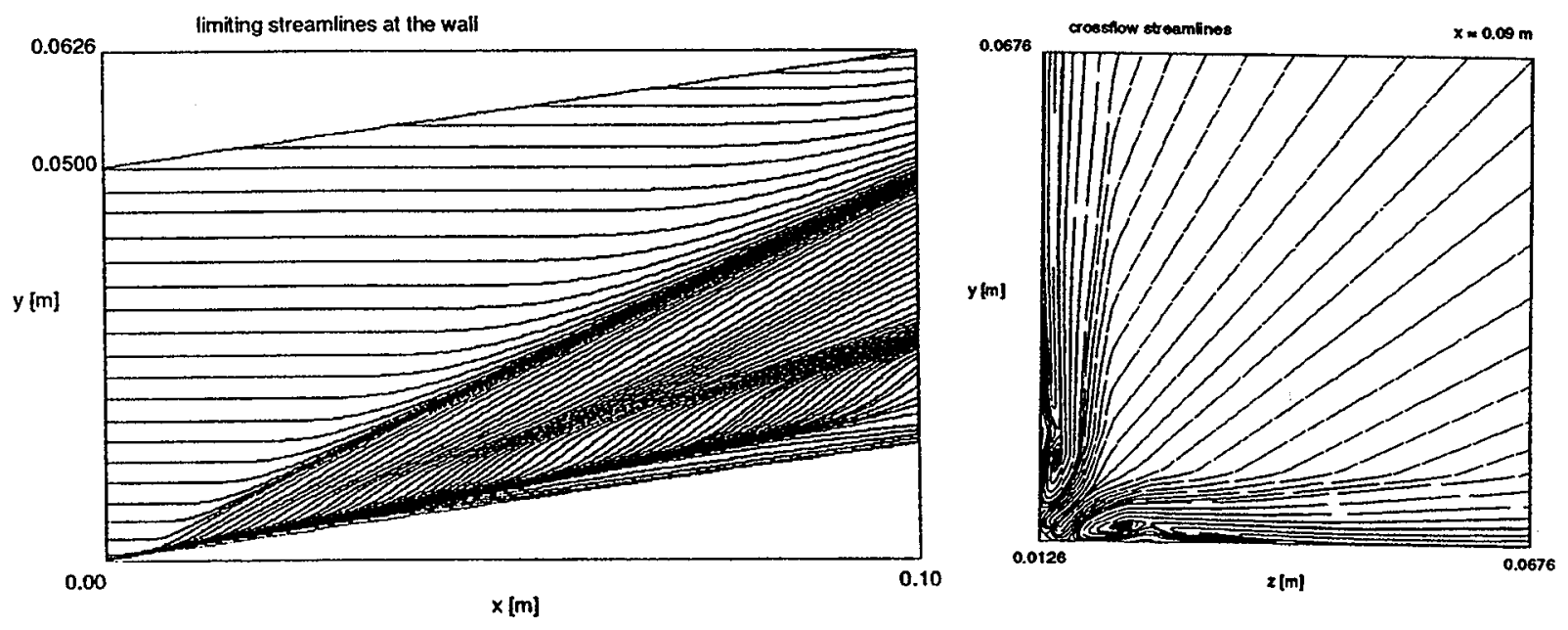

Figure 13: Limiting streamlines at the wail and crossflow streamlines for test case \#1.

value should be $3.3^{\circ}$ ). Nevertheless, the maximum Pitot pressure value is 6.70 for the computation, and experiments show their highest value on the contour corresponding to 6.42 .

In addition, in figure 15a, numerical and experimental static pressures at the wall are overlaid, showing a satisfactory agreement. Here, as in the following, static pressures at the wall have been adimensioned with $p_{K}$, which is the pressure on a $2 \mathrm{D}$ ramp with the same slope of the wedge. In figure $15 \mathrm{c}$, the limiting flow direction at the wall is compared. The two curves are very similar close to the corner, as demonstrated by the fact that the reattachment of the primary vortex is detected in the same position (note that $\psi=0$ indicates the presence of a separation or of a reattachment). Nevertheless, receding from the intersection of the ramps, some not negligible differences appear. In the computation, the secondary vortex is smaller and closer to the corner (though not in such a great extent) and the separation of the primary vortex is located in a different position with respect to the experiments. Such discrepancies might possibly be due to the fact that, approaching the lateral boundaries of the model, experiments could be affected by side-effects or by interference with the tunnel boundary layer. On the other hand, it should be also recognized the extreme sensitivity of the flow structure to the upstream Mach number and to the uncertainty about its level and its uniformity along the tunnel axis [42]. Finally, in figure $15 \mathrm{~b}$, the heat flux at the wall is compared. As it could be expected after the previous discussion, results are similar as far as the reattachment of the primary vortex is concerned, with the peak of heat transfer in the same position and showing a similar magnitude. Conversely, the location of the peak related to the secondary vortex is different, though the value is the same. Other discrepancies concern the fact that, in the numerical results, we find no trace of the peak which, in the experimental curve, is signaled at $\bar{y} \simeq 0.09$. Last, it can be noticed that the computation predicts an almost null heat flux locally at the corner, in contrast with the experiments; in this case we think it is reasonable to agree with the numerical result, since very close to the corner 


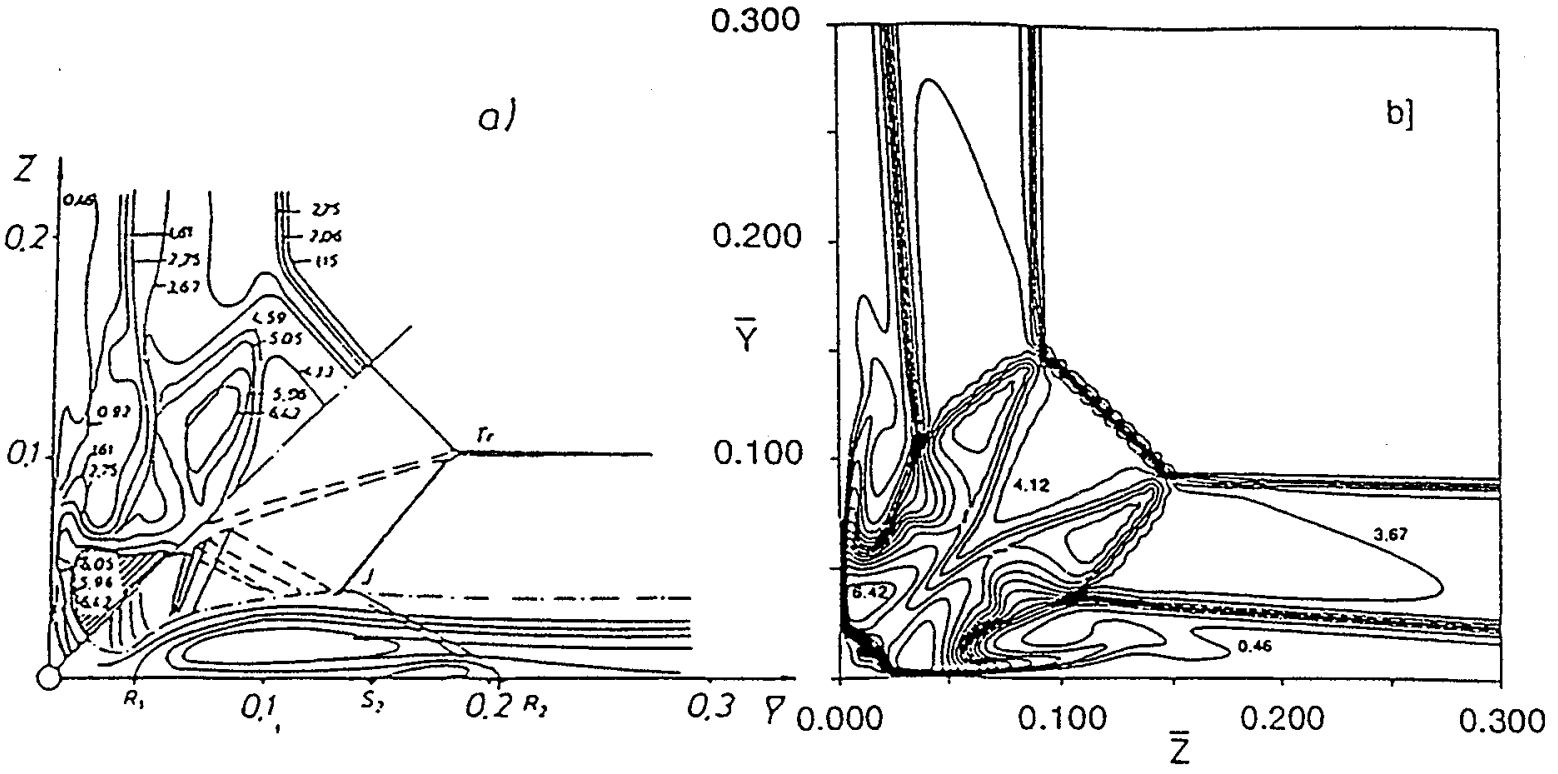

Figure 14: Pitot pressure contours for test case \#1: (a)experiment [28], (b)computation.

the temperature varies very smoothly, and on the other hand the measurement technique used cannot approach too much to the corner itself.

In order to adequately address the accuracy of the computation, the results obtained with the $100 \times 100$ grid are compared with those resulting from a $60 \times 60$ grid. The main features of the flowfield are unchanged, as it can be seen from figure 16, where pitot pressure contours are shown. The major difference is related to the secondary vortex: with the finer mesh it is fully captured, while with the coarser one its presence is just sensed. A proof to this statement can be found in figures 17 and 18. In figure 17, crossflow streamlines are presented: in the left picture $(60 \times 60$ mesh $)$, the undulation of the crossflow streamlines suggests the presence of the secondary vortex, but in the right one $(100 \times 100$ mesh), the vortex is well evident. In figure 18, the flow direction at the wall is shown again: it can be seen that in the case of the finer mesh $(100 \times 100)$, a crossflow reversal is present at about $\bar{y}=0.12$, while with the coarser mesh $(60 \times 60)$ this feature does not exist.

Test case \#2 is characterized by a stronger viscous interaction with respect to test case \#1, since the Mach number is greater and the Reynolds number is lower. Thus, a thicker viscous layer and a thinner inviscid portion of the shock layer are expected. Such features are shown in figures 19 and 20, where crossflow streamlines and Mach number contours are shown. In this occasion, the secondary separation has not been captured by numerical tests, and also the experimental data that will be shown in the following do not help to understand if it exists or not. Investigations of the flow direction at the wall, reported in figure 21 , show that the limiting streamlines below the primary vortex tend to deviate as if a secondary separation was incipient but still not developed, though it is not clear whether this is the real physical picture or if a finer grid would reveal the effective presence of small vortex there. It is interesting to note that, in any case, the primary separation is again constituted of two foci and one saddle, a configuration that is topologically possible also without the presence of the secondary embedded vortex.

In figures $22 \mathrm{a}$ and $22 \mathrm{~b}$, Pitot-pressure contours at $\mathrm{x}=0.09 \mathrm{~m}$ are compared, showing a good agreement. In figure $22 \mathrm{c}$, the pressure distribution at the wall is shown. Finally, in figure $22 \mathrm{~d}$, heat fluxes at the wall are compared; the agreement is good, though, as before, there is a local difference at the corner and a second peak in the experimental results. The latter 
Pw is the pressure for the inviscid 2D wedge

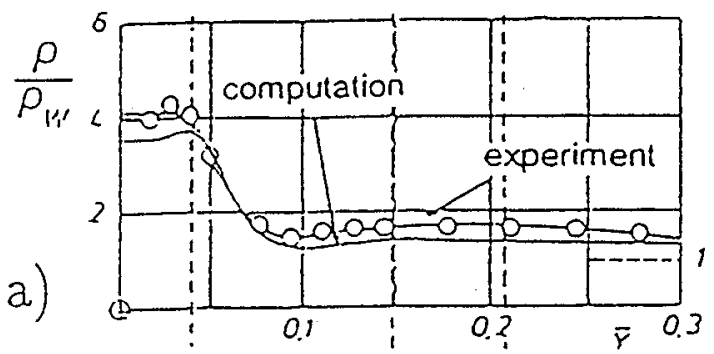

$Q_{w}$ is the heat Bux at the wall for the $2 D$ wedge

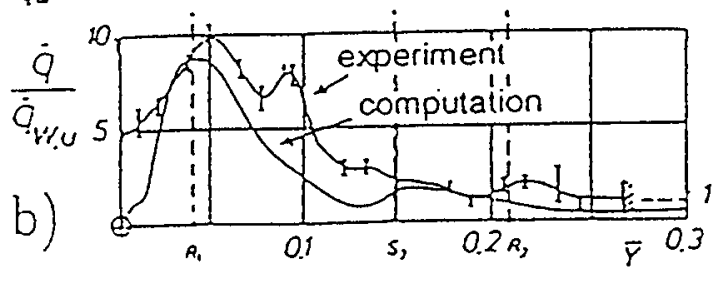

$\bar{y}=\frac{\nu}{x}-\operatorname{tg} \delta \quad \begin{aligned} & \psi \text { is angle between wall streamities } \\ & \text { and tbe conical dircction }\end{aligned}$

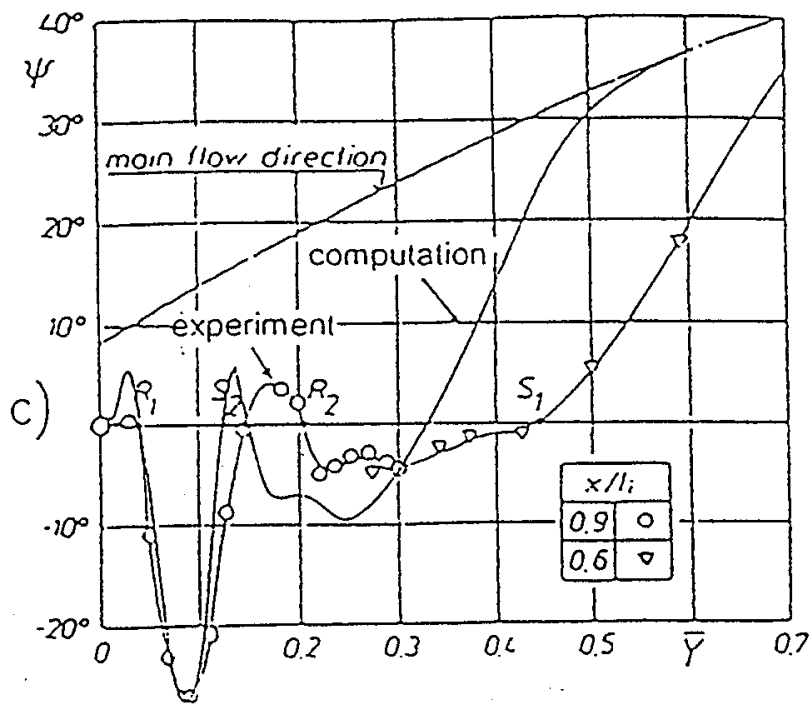

Figure 15: Wall pressure (a), heat fluxes (b) and flow direction (c) at the wall: experiments [28] and computations.

feature may be due to the presence of a region of high speed flow underneath the primary vortex, where very steep total pressure gradients exist and which is possibly not properly described by the numerics. It should be also noticed that no secondary reattachment (and therefore also separation) seems to be shown by experimental measurements.

Test case \#3 is characterized by a corner angle of $120^{\circ}$, while the remaining geometrical and freestream conditions are those of test case \#2. As, increasing the corner angle, the embedded shocks weaken and pressure gradients are in general smaller, then also the flow separation is less intense with respect to a corner of $90^{\circ}$. The greater amplitude of the corner angle determines a lower pressure level in the corner region, so that vortical structures are more inboard. From the crossflow streamlines displayed in figure 24, it possible to see that also in this case the secondary separation is not present, though the primary vortex is again splitted in two parts. Moreover, plots of the flow direction at the wall do not indicate neither an incipient separation.

A comparison with experimental results is made in figure 23: the agreement seems to be good, though the presence of the second peak can be noticed in the experimental heat transfer measurements, but not in the numerical results.

\subsection{The effect of the local Reynolds number}

Some numerical experiments have been performed on test case \#1 to investigate the effect of the local Reynolds number $R e_{x}$ on the conicity of the flow and on the development of shock-induced vortical structures. Thus, the same upstream flow conditions and different lengths of the corner configuration will be assumed. At the highest Reynolds numbers the flow is likely to be turbulent and therefore the corresponding results presented here are not realistic. Nevertheless, it was decided to show them just to demonstrate how the flowfield is modified depending whether viscous or inviscid effects dominate. 

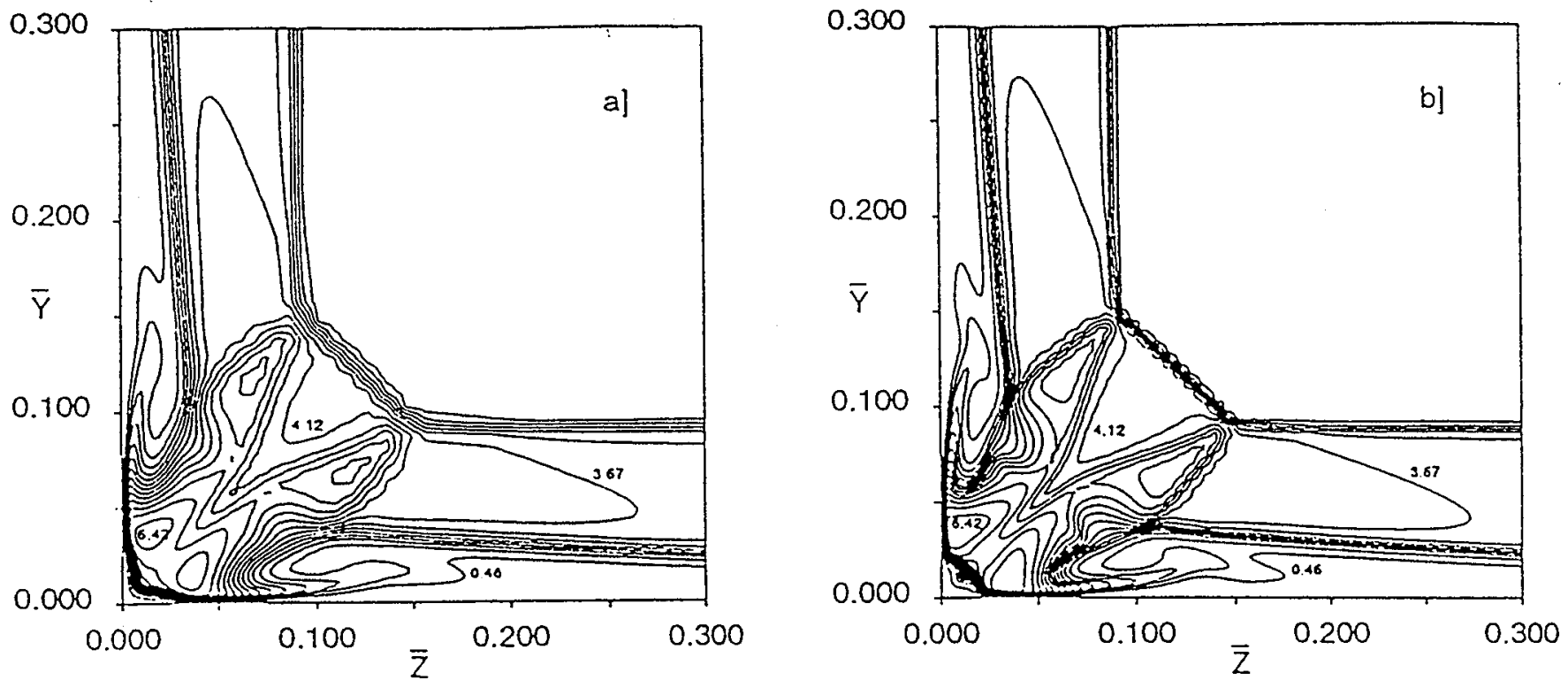

Figure 16: Pitot pressure contours computed with different meshes: (a)60x60 mesh, (b) $100 \times 100$ mesh.

In figure 25, wall pressure distributions corresponding to different local Reynolds numbers have been plotted. Pressures have been dimensioned using the corresponding inviscid value and distances using the distance $x$ from the leading edge. If the flow was conical, the six graphs shown here should overlay, but this is not the case. It should be noticed that test case \#1 is characterized by a hypersonic freestream Mach number. Thus, viscous interaction effects are rather strong, as evidenced by the graphs, that show that wall pressure distributions far from the corner, where the flow is essentially two-dimensional, are higher, for low local Reynolds numbers, than the inviscid value. Therefore, the whole flowfield is not conical as long as strong viscous interaction effects are present.

Moreover, also the structure of the vortex system seems to change with increasing the distance from the leading edge. For low local Reynolds numbers, the flow is dominated by transport phenomena. Thus, the viscous layer is very thick and occupies a large portion of the shock layer (figures 26 and 27). According to numerical results, only the primary separation is present, splitted in two parts as usual (figure 28). Proceeding to move downstream, the secondary separation appears, as already shown in figure 12 . For even larger local Reynolds numbers (that in effect are likely to correspond to turbulent conditions), the primary separation appears to be composed of three foci (figure 29), and the embryo of the vortical structures that characterize the Euler solution [32] can be perceived close to the symmetry line (figure 30 ).

\section{Conclusions}

Despite the simplicity of their geometry, supersonic corner configurations represent a rather complex fluid dynamic test case, as, owing to the mutual interaction between inviscid and viscous effects, important vortical structures that strongly characterize the 

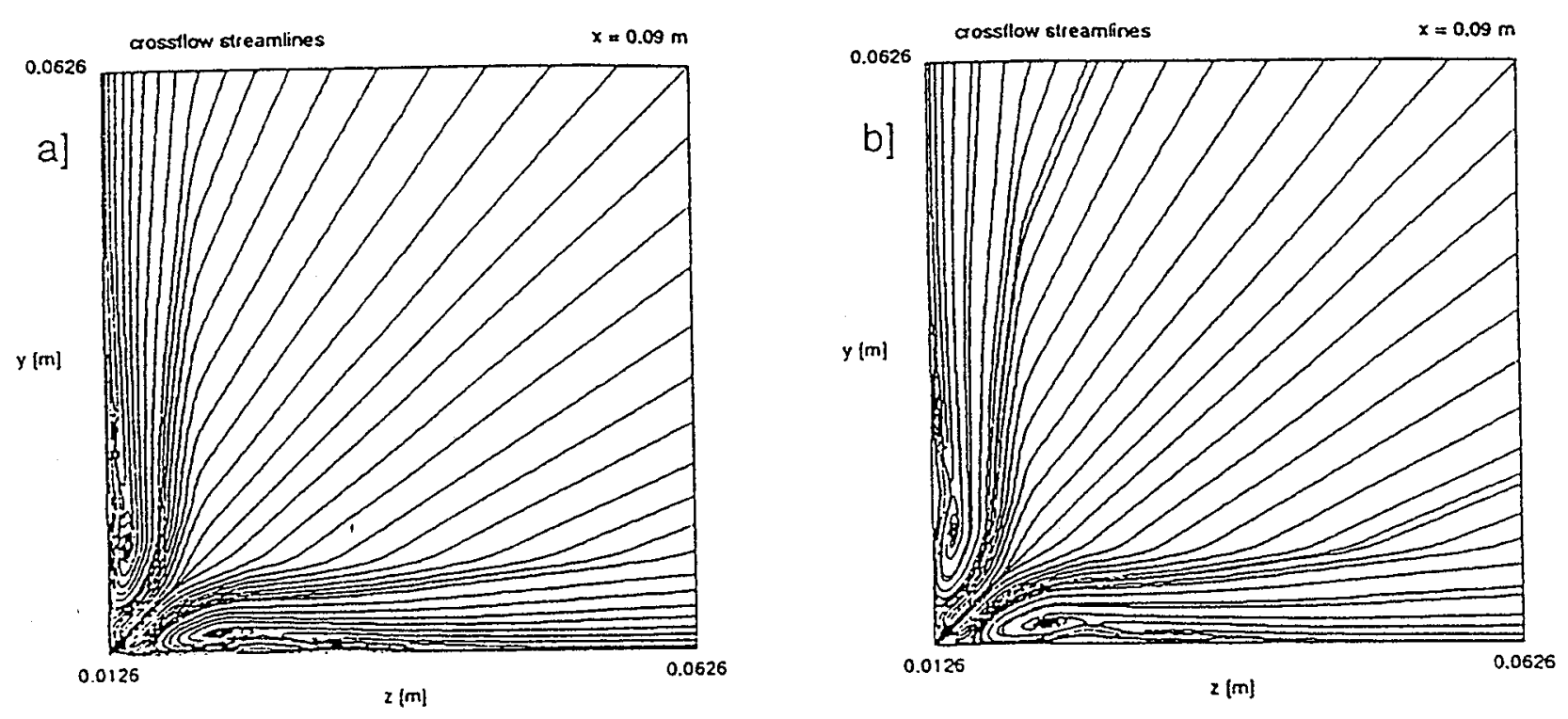

Figure 17: Computed crossflow streamlines: (a)60x60 mesh, (b)100x100 mesh.

flowfield are created. Since the very first studies, that started about 35 years ago, the most important flowfield features are, at this time, known and understood. Concerning the laminar regime, however, it is still not very clear which is the sensitivity of the flowfield features to the geometrical and upstream conditions, that seem to influence very much the extension and the complexity of the vortical structures. It is the hope of the authors to have suggested, through this paper, that the computational fluid dynamics, strictly tied to experimental tests in a process of mutual validation, could be considered as a very useful tool to conduct such studies in a reasonable time.

\section{References}

[1] J. Sternberg, "Triple-shock-wave intersections," The Physics of Fluids, vol. 2, pp. 179-206, March-April 1959.

[2] F. Marconi, "Supersonic, inviscid, conical corner flowfields," AIAA Journal, vol. 18, January 1980.

[3] F. Marconi, The Supersonic Flow in Conical Corners. PhD thesis, Polytechnic Institute of New York, 1981.

[4] M. H. Bloom and S. Rubin, "High-speed viscous corner flow," J. of Aerospace Sciences, vol. 28, February 1961.

[5] P. A. Libby, "Secondary flows associated with a supersonic corner region," $A I A A$ Journal, vol. 4, April 1966.

[6] P. C. Stainback, "An experimental investigation at a mach number of 4.95 of flow in the vicinity of a $90^{\circ}$ interior corner alined with a free-stream velocity," Technical Note D-184, NASA, 1960. 
[7] P. C. Stainback, "Heat-transfer measurements at a mach number of 8 in the vicinity of a $90^{\circ}$ interior corner alined with a free-stream velocity," Technical Note D-2417, NASA, 1964.

[8] F. D. Hains, "Supersonic flow near the junction of two wedges," J. of Aerospace Sciences, vol. 25, April 1958.

[9] J. Wallace and J. h. Clarke, "Uniformly valid second-order solution for supersonic flow over cruciform surfaces," AIAA Journal, vol. 1, January 1963.

[10] S. M. Bogdonoff and I. E. Vas, "A preliminary investigation of the flow in a $90^{\circ}$ corner at hypersonic speeds. part $\mathrm{i}$ - flat plates with thin leading edges at zero angle of attack," Technical Report D143-978-013 (ARDC TR 57-2-2, AD 150 023), Bell Aircraft Corp., 1957.

[11] A. F. Charwat and L. G. Redekeopp, "Supersonic interference flow along the corner of intersecting wedges," AIAA Journal, vol. 5, March 1967.

[12] P. C. Stainback and L. M. Weinstein, "Aerodynamics heating in the vicinity of corners at hypersonic speeds," Technical Note D-4130, NASA, 1967.

[13] R. J. Cresci, S. G. Rubin, C. T. Nardo, and T. C. Lin, "Hypersonic interaction along a rectangular corner," AIAA Journal, vol. 7, December 1969.

[14] R. D. Watson and L. M. Weinstein, "A study of hypersonic corner flow interaction," AIAA Journal, vol. 9, July 1971.

[15] J. W. Keyes and R. D. Watson, "Laminar heating in interior corners at Mach 19," J. Spacecraft, vol. 7, August 1970.

[16] J. E. West and R. H. Korkegi, "Supersonic interaction in the corner of intersecting wedges at high Reynolds number," AIAA Journal, vol. 10, May 1972.

[17] R. D. Watson," "Experimental study of sharp- and blunt-nose streamwise corners at mach 20," Technical Note D-7398, NASA, 1974.

[18] J. R. Cooper and W. L. Hankey, "Flowfield measurement in an asymmetric axial corner at $\mathrm{M}=12.5, "$ AIAA Journal, vol. 12, October 1974.

[19] K. Kipke and D. Hummel, "Untersuchungen an längsangeströmten Eckenkonfigurationen im Hyperschallbereich. Teil I: Ecken zwischen ungepfeilten Keilen," Z. Flugwiss., vol. 23, Dezember 1975.

[20] R. H. Korkegi, "On the structure of three-dimensional shock-induced separated flow regions," AIAA Journal, vol. 14, May 1976.

[21] P. Kutler, "Supersonic flow in the corner formed by two intersecting wedges," AIAA Journal, vol. 12, May 1974.

[22] V. Shankar, D. Anderson, and P. Kutler, "Numerical solutions for supersonic corner flows," Journal of Computational Physics, vol. 17, 1975. 
[23] D. A. Anderson and R. K. Nangia, "Comparison of numerical and experimental conical flow fields in supersonic corners with compression and/or expansion," Aeronautical Quaterly, vol. 28, November 1977.

[24] J. S. Shang and W. L. Hankey, "Numerical solution of the Navier-Stokes equations for a three-dimensional corner," AIAA Journal, vol. 15, November 1977.

[25] C. M. Hung and R. W. MacCormack, "Numerical solutions of three-dimensional shock wave and turbulent boundary layer interaction," (Huntsville, Alabama), 1978. AIAA Paper 78-161.

[26] J. S. Shang, W. L. Hankey, and J. S. Petty, "Three-dimensional supersonic interacting turbulent flow along a corner," AIAA Journal, vol. 17, July 1979.

[27] W. Möllenstädt, "Untersuchungen an längsangeströmten Eckenkonfigurationen im Hyperschallbereich. Teil II: Ecken zwischen gepfeilten Keilen," Z. Flugwiss. Weltraumforsch, vol. 8, no. 6, 1984.

[28] D. Hummel, "Experimental investigations on blunt bodies and corner configurations in hypersonic flow," in Aerodynamics of hypersonic lifting vehicles, 1987. AGARDCP-428, paper 6.

[29] N. Qin, K. W. Scriba, and B. E. Richards, "Shock-shock, shock-vortex interaction and aerodynamic heating in hypersonic corner flow," Aeronautical Journal, May 1991.

[30] G. Degrez, "Swept shock wave/laminar boundary layer interactions, experimental and numerical results," in Special course on shock-wave/boundary-layer interactions in supersonic and hypersonic flows, 1993. AGARD-R-792, paper 2.

[31] H. Petzel and D. Hummel, "Experimentelle untersuchung einer langsdurchstromten unsymmetrichen ecke bei $m_{\infty}=12.6, "$ Jahrbuch 1992 der Deutschen Gesellshaft fur Luft and Raumfahrt, 1992.

[32] R. Marsilio, "Vortical solutions in supersonic corner flows," AIAA Journal, vol. 31, September 1993.

[33] G. Degrez, P. Spazzini, R. Marsilio, and M. Pandolfi, "Asymmetric vortical solutions in supersonic corners: Steady 3-d space-marching versus time-dependent conical results," in AIAA 27th Fluid Dynamics Conference, (Orlando, Florida), 1993. AIAA Paper 93-2957.

[34] Y. C. Vigneron, J. V. Rakich, and J. C. Tannehill, "Calculation of supersonic viscous flow over delta wings with sharp subsonic leading edges," Technical Memorandum 78500, NASA, June 1978.

[35] D. A. Anderson, J. C. Tannehill, and R. H. Pletcher, Computational Fluid Mechanics and Heat Transfer, ch. 8. New York: McGraw-Hill Book Company, 1984.

[36] J. J. Korte, "An explicit upwind algorithm for solving the Parabolized Navier-Stokes equations," Technical Paper 3050, NASA, February 1991. 
[37] J. H. Morrison and J. J. Korte, "Implementation of Vigneron's streamwise pressure gradient approximation in the PNS equations,". in AIAA 30th Aerospace Sciences Meeting and Exhibit, (Reno, Nevada), Jan. 6-9 1992. AIAA Paper 92-0189.

[38] D. D'Ambrosio and R. Marsilio, "A numerical method for solving the three-dimensional Parabolized Navier-Stokes equations," ICASE Report No. 95-38, ICASE NASA Langley Research Center, 1995.

[39] M. Pandolfi, "Computation of steady supersonic flows by a flux difference splitting method," Journal of Computers and Fluids, vol. 10, no. 1, 1985.

[40] A. Harten, B. Engquist, S. Osher, and S. R. Chakravarthy, "Uniformly high order accurate Essentially Non-Oscillatory schemes, III," Journal of Computational Physics, vol. $71,1987$.

[41] M. Germano, "Topology of surface shear-stress lines," Arch. Mech., vol. 34, pp. 605$619,1982$.

[42] D. Hummel, "Private communication," April 1995. 


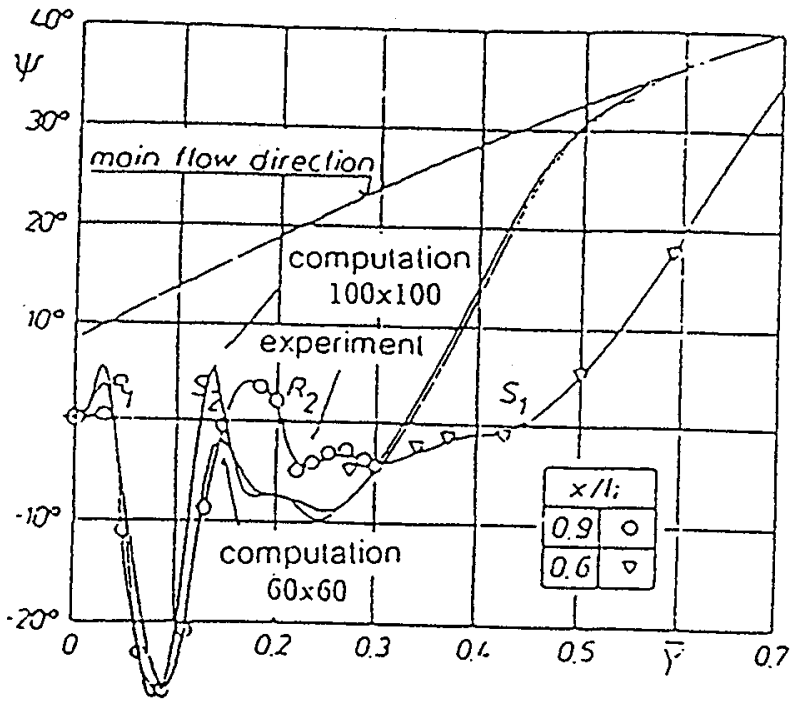

Figure 18: Flow direction at the wall: experiments [28], $60 \times 60$ mesh and 100x100 mesh.

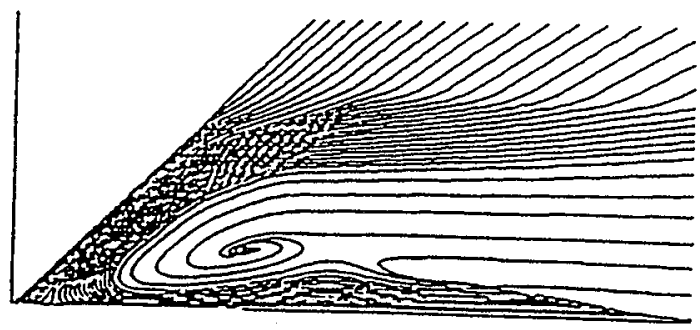

Figure 19: Computed crossflow streamlines for test case \#2.

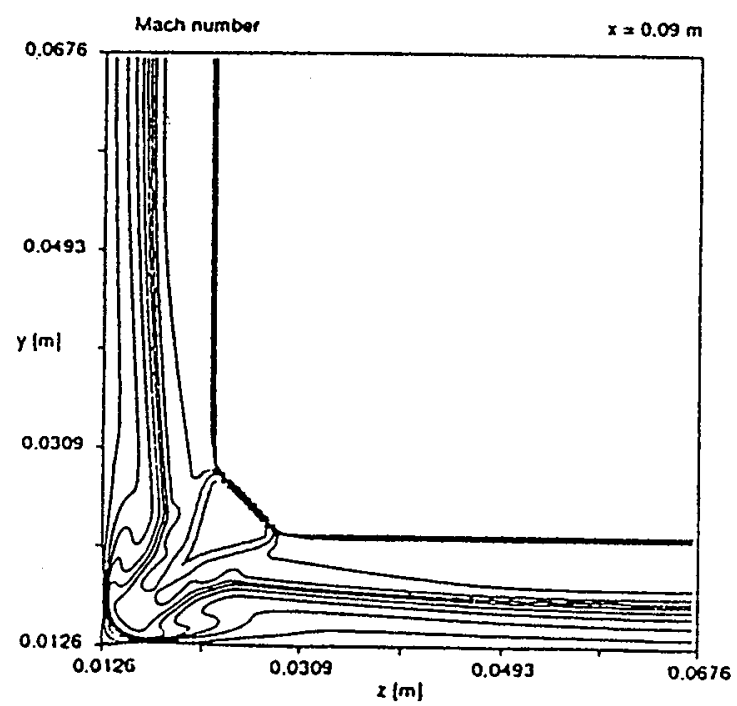

Figure 20: Computed Mach number contours for test case \#2. 


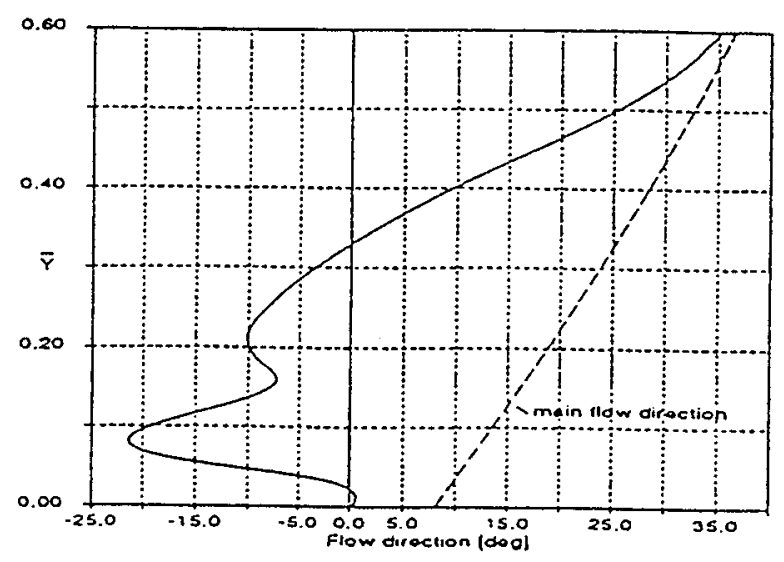

Figure 21: Test case\#2: flow direction at the wall.
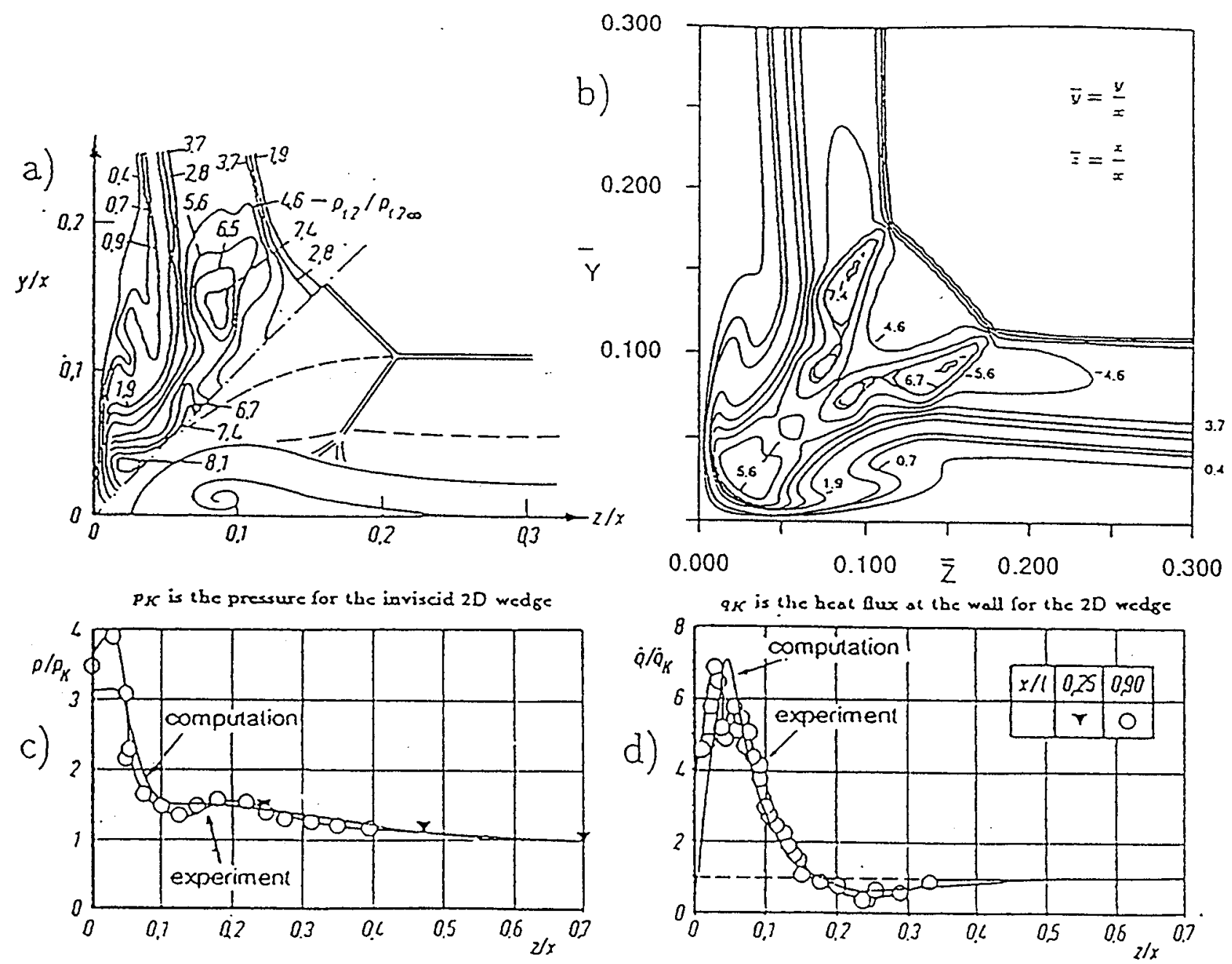

Figure 22: Test case \#2. Pitot pressure contours: (a)experiment [19], (b)computation. Wall: (c)pressure, (d) heat transfer. 

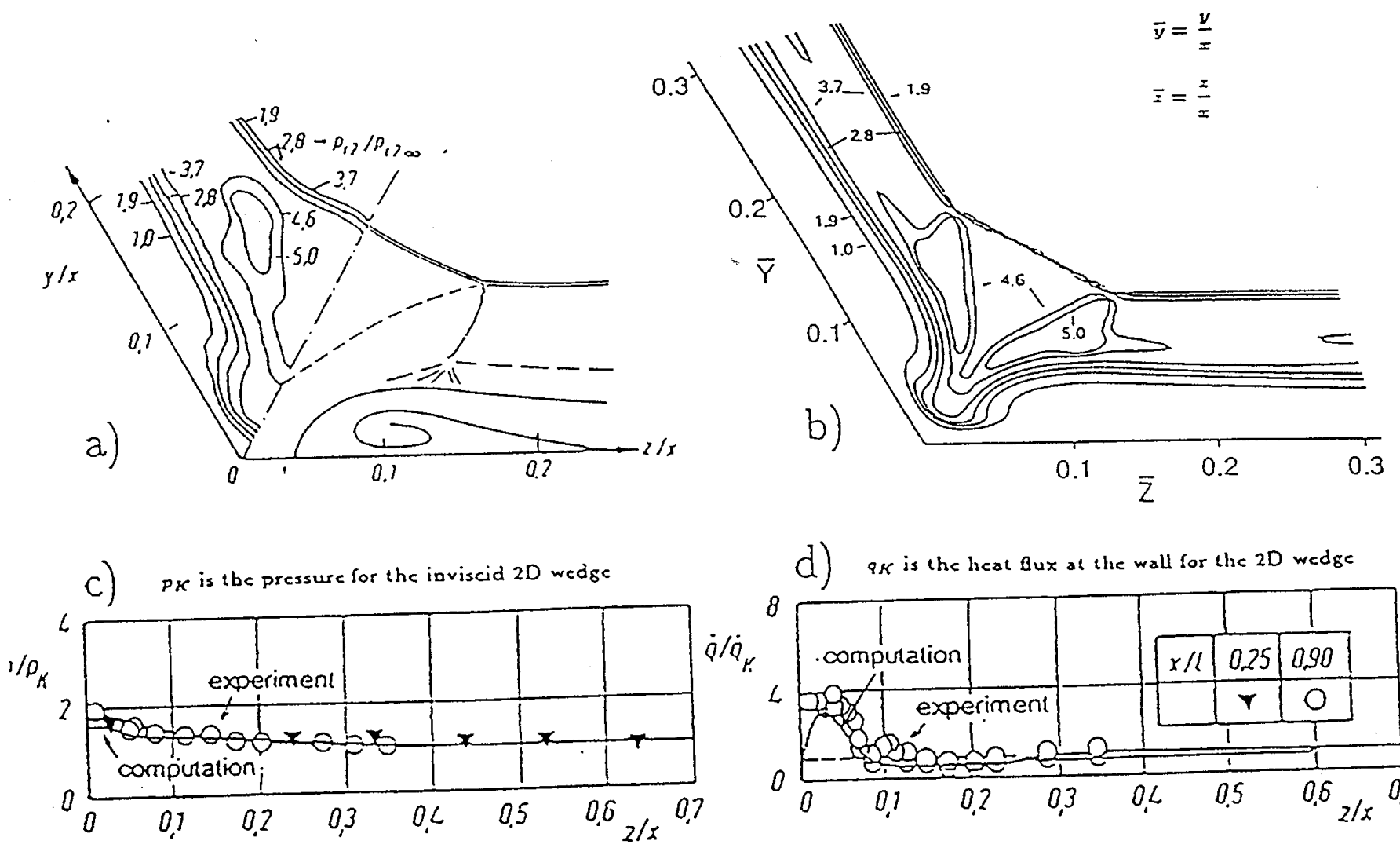

d) $9 K$ is the heat Bux at the wall for the 2D wadge

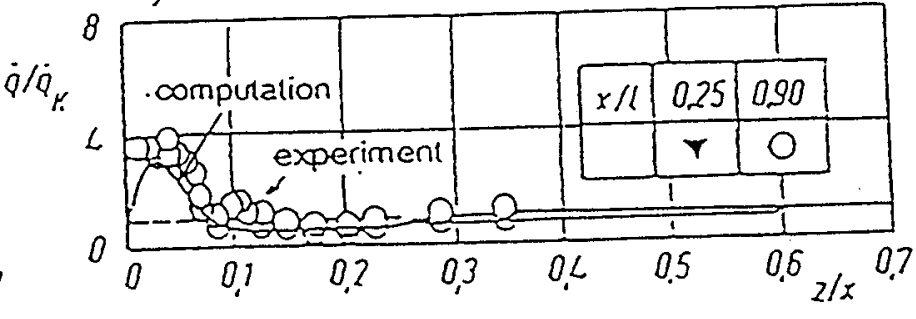

Figure 23: Test case \#3. Pitot pressure contours: (a)experiment[19], (b)computation. Wall: (c)pressure, (d) heat transfer.

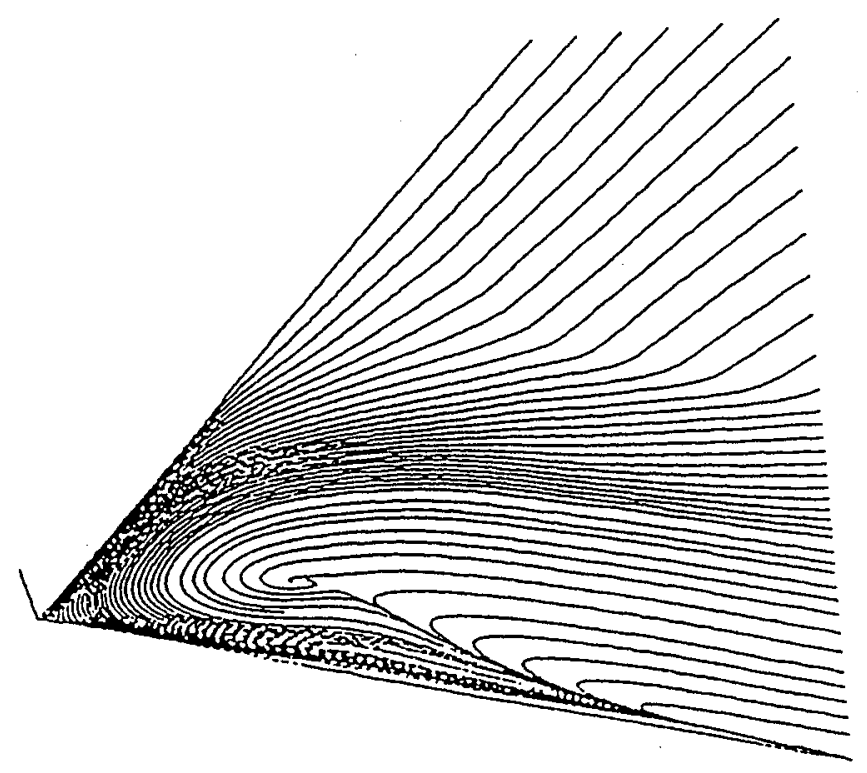

Figure 24: Computed crossflow streamlines for test case \#3. 


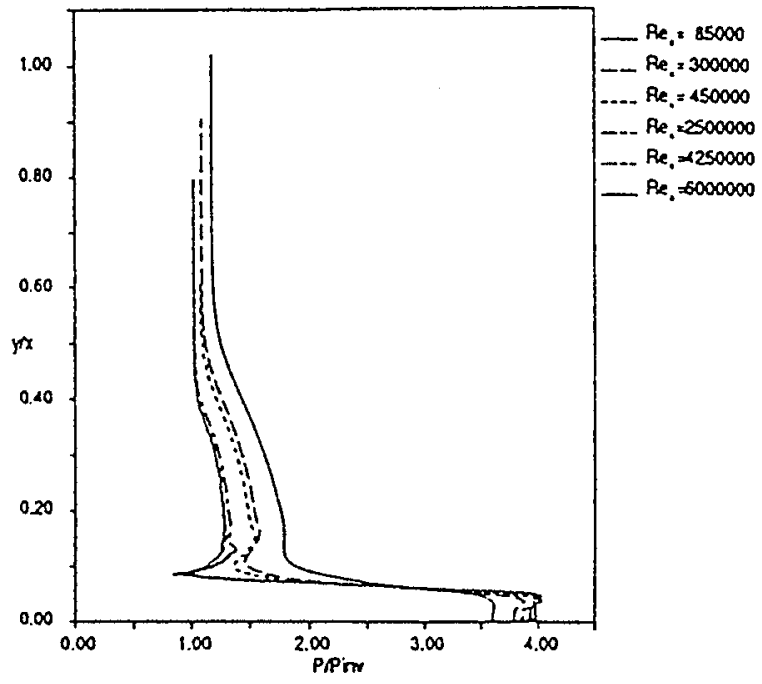

Figure 25: Test case \#1: wall pressure distributions for different local Reynolds numbers.

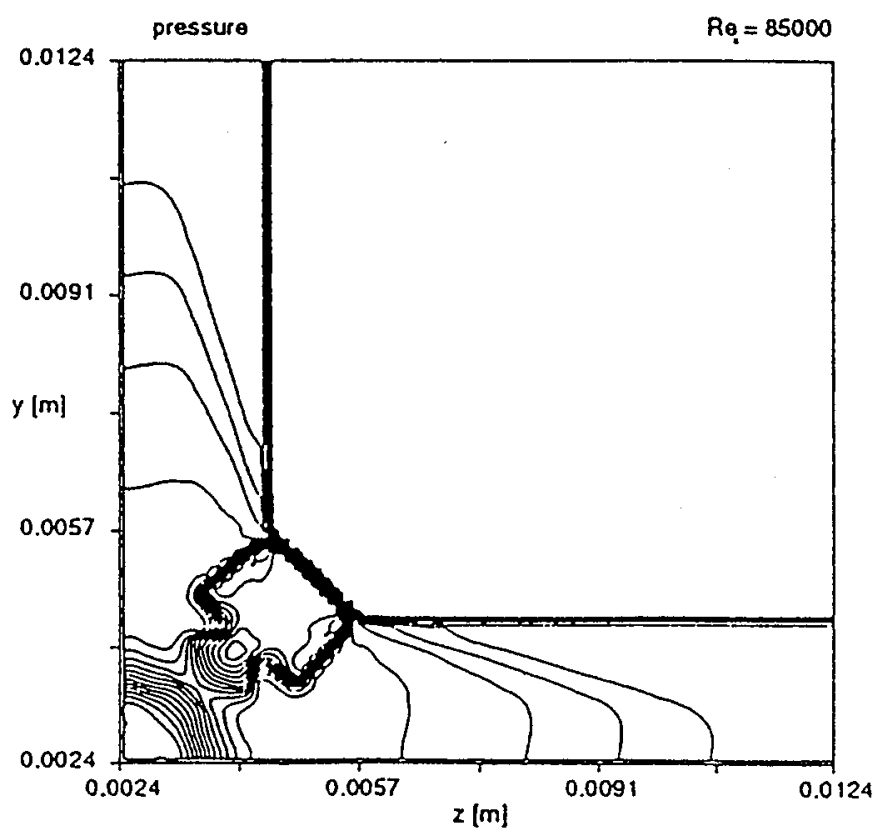

Figure 26: Computed pressure contours for test case \#1 at $R e_{x}=85000$. 


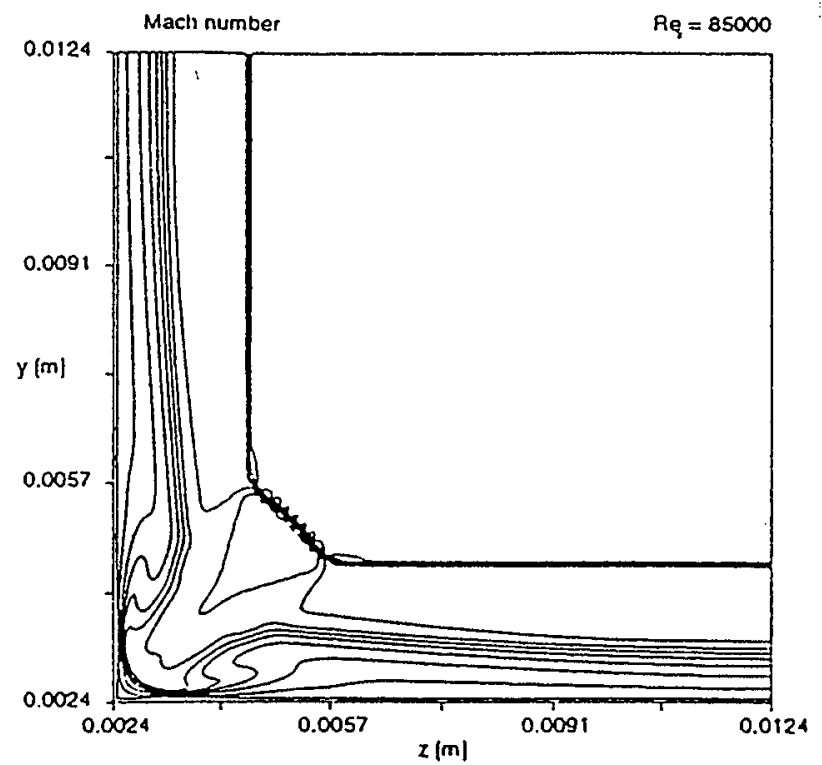

Figure 27: Computed Mach number contours for test case \#1 at $R e_{x}=85000$.

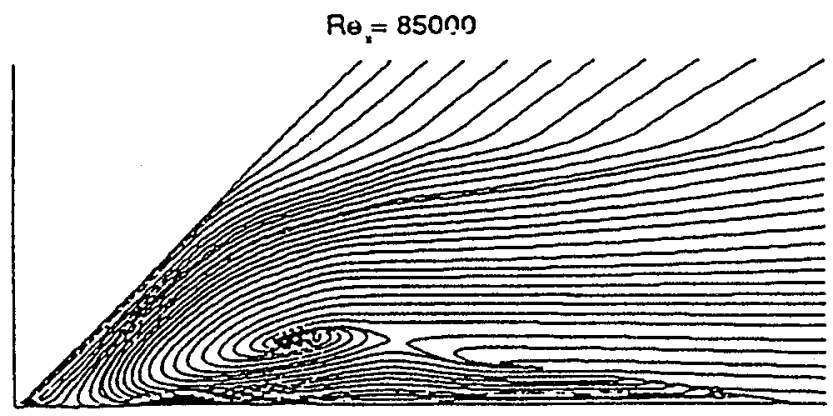

Figure 28: Computed crossflow streamlines for test case \#1 at $R e_{x}=85000$.

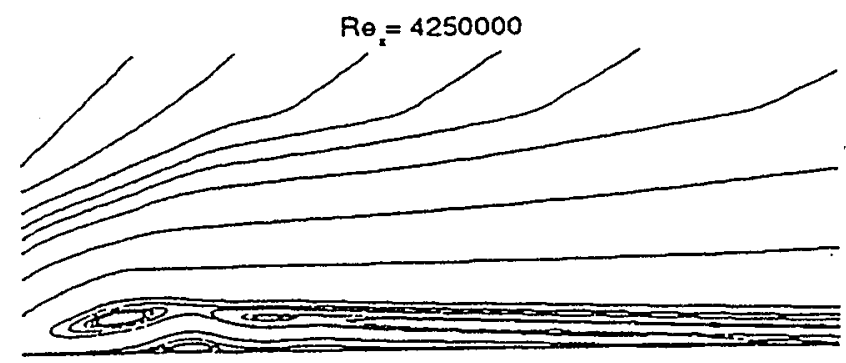

Figure 29: Computed crossflow streamlines for test case \#1 at $R e_{x}=4250000$. 


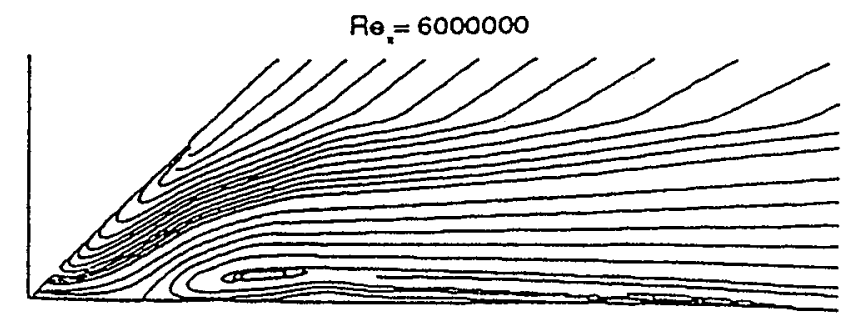

Figure 30: Computed crossflow streamlines for test case $\# 1$ at $R e_{x}=6000000$. 



\begin{tabular}{|c|c|c|c|}
\hline \multicolumn{3}{|c|}{ REPORT DOCUMENTATION PAGE } & $\begin{array}{l}\text { Form Approved } \\
\text { OMB No. 0704-0188 }\end{array}$ \\
\hline \multicolumn{4}{|c|}{ 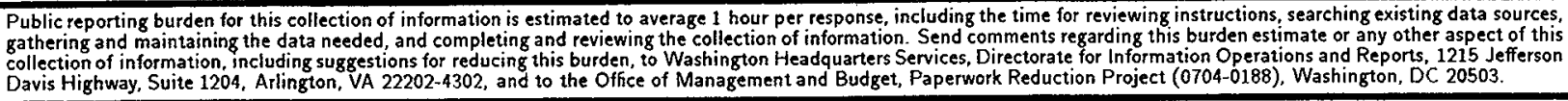 } \\
\hline 1. AGENCY USE ONLY (Leave blank) & $\begin{array}{l}\text { 2. REPORT DATE } \\
\text { December } 1995\end{array}$ & \multicolumn{2}{|c|}{$\begin{array}{l}\text { 3. REPORT TYPE AND DATES COVERED } \\
\text { Contractor Report }\end{array}$} \\
\hline \multicolumn{3}{|c|}{$\begin{array}{l}\text { 4. TITLE AND SUBTITLE } \\
\text { SHOCK-INDUCED SEPARATED STRUCTURES IN } \\
\text { SYMMETRIC CORNER FLOWS }\end{array}$} & \multirow[t]{2}{*}{$\begin{array}{l}\text { 5. FUNDING NUMBERS } \\
\text { C NAS1-19480 } \\
\text { WU } 505-90-52-01\end{array}$} \\
\hline \multicolumn{3}{|l|}{$\begin{array}{l}\text { 6. AUTHOR(S) } \\
\text { Domenic D'Ambrosio } \\
\text { Roberto Marsilio }\end{array}$} & \\
\hline \multicolumn{3}{|c|}{$\begin{array}{l}\text { 7. PERFORMING ORGANIZATION NAME(S) AND ADDRESS(ES) } \\
\text { Institute for Computer Applications in Science and Engineering } \\
\text { Mail Stop 132C, NASA Langley Research Center } \\
\text { Hampton, VA 23681-0001 }\end{array}$} & $\begin{array}{l}\text { 8. PERFORMING ORGANIZATION } \\
\text { REPORT NUMBER } \\
\text { ICASE Report No. } 95-79\end{array}$ \\
\hline \multicolumn{3}{|c|}{$\begin{array}{l}\text { 9. SPONSORING/MONITORING AGENCY NAME(S) AND ADDRESS(ES) } \\
\text { National Aeronautics and Space Administration } \\
\text { Langley Research Center } \\
\text { Hampton, VA 23681-0001 }\end{array}$} & $\begin{array}{l}\text { 10. SPONSORING/MONITORING } \\
\text { AGENCY REPORT NUMBER } \\
\text { NASA CR-198239 } \\
\text { ICASE Report No. } 95-79\end{array}$ \\
\hline \multicolumn{4}{|c|}{$\begin{array}{l}\text { 11. SUPPLEMENTARY NOTES } \\
\text { Langley Technical Monitor: Dennis M. Bushnell } \\
\text { Final Report } \\
\text { To be submitted to Journal of Fluid Mechanics }\end{array}$} \\
\hline \multicolumn{2}{|c|}{$\begin{array}{l}\text { 12a. DISTRIBUTION/AVAILABILITY STATEMENT } \\
\text { Unclassified-Unlimited } \\
\text { Subject Category } 34\end{array}$} & & 12b. DISTRIBUTION CODE \\
\hline \multicolumn{4}{|c|}{$\begin{array}{l}\text { 13. ABSTRACT (Maximum } 200 \text { words) } \\
\text { Three-dimensional supersonic viscous laminar flows over symmetric corners are considered in this paper. The } \\
\text { characteristic features of such configurations are discussed and an historical survey on the past research work is } \\
\text { presented. A new contribution based on a numerical technique that solves the parabolized form of the Navier-Stokes } \\
\text { equations is presented. Such a method makes it possible to obtain very detailed descriptions of the flowfield with } \\
\text { relatively modest CPU time and memory storage requirements. The numerical approach is based on a space-marching } \\
\text { technique, uses a finite volume discretization and an upwind flux-difference splitting scheme (developed for the steady } \\
\text { flow equations) for the evaluation of the inviscid fluxes. Second order accuracy is reached following the guidelines } \\
\text { of the ENO schemes. Different free-stream conditions and geometrical configurations are considered. Primary and } \\
\text { secondary streamwise vortical structures embedded in the boundary layer and originated by the interaction of the } \\
\text { latter with shock waves are detected and studied. Computed results are compared with experimental data taken } \\
\text { from literature. }\end{array}$} \\
\hline \multirow{2}{*}{\multicolumn{3}{|c|}{$\begin{array}{l}\text { 14. SUBJECT TERMS } \\
\text { CFD; Parabolized Navier-Stokes Equations; Intersection Shock; } \\
\text { Boundary Layer; Vortex Flows }\end{array}$}} & $\begin{array}{l}\text { 15. NUMBER OF PAGES } \\
32 \\
\end{array}$ \\
\hline & & & $\begin{array}{r}\text { 16. PRICE CODE } \\
\mathrm{A} 03\end{array}$ \\
\hline $\begin{array}{l}\text { 17. SECURITY CLASSIFICATION } \\
\text { OF REPORT } \\
\text { Unclassified }\end{array}$ & $\begin{array}{l}\text { 18. SECURITY CLASSIFICATION } \\
\text { OF THIS PAGE } \\
\text { Unclassified }\end{array}$ & $\begin{array}{l}\text { 19. SECURITY CLASSIFICATION } \\
\text { OF ABSTRACT }\end{array}$ & $\begin{array}{l}\text { 20. LIMITATION } \\
\text { OF ABSTRACT }\end{array}$ \\
\hline NSN 7540-01-280-5500 & & & $\begin{array}{l}\text { Standard Form 298(Rev. 2-89) } \\
\text { Prescribed by ANSI Std. Z39-18 } \\
298-102\end{array}$ \\
\hline
\end{tabular}


